\title{
Generalized Flory equations of state for copolymers modeled as square-well chain fluids
}

\author{
Harpreet S. Gulati and Carol K. Hall \\ Department of Chemical Engineering, Box 7905, North Carolina State University, \\ Raleigh, North Carolina 27695-7905
}

(Received 3 July, 1997; accepted 27 January 1998)

\begin{abstract}
The Generalized Flory-Dimer (GFD) equation of state has been extended to fluids containing copolymers modeled as heteronuclear chains of freely-jointed tangent spheres that interact via a site-site square-well potential. Compressibility factors are obtained for block, alternating and random copolymer fluids. The GFD theory for square-well copolymers requires expressions for the insertion factors of SW monomers in SW monomer mixtures, SW dimers in SW dimer mixtures, and SW heteronuclear dumbbells in SW heteronuclear dumbbell fluids. These insertion factors are obtained using recently-derived perturbation-theory-based equations of state. The effects of variations in composition, segment size ratios, and well-depth ratios on the compressibility factor are studied. The predictions of the Generalized Flory-Dimer theory are compared to compressibility factors obtained from discontinuous canonical molecular dynamics simulation. The Generalized Flory-Dimer theory accurately predicts the compressibility factors of square-well copolymer fluids for a variety of cases including those in which the size ratio and well-depth ratio of the two components are different. (C) 1998 American Institute of Physics. [S0021-9606(98)50617-4]
\end{abstract}

\section{INTRODUCTION}

Fluids containing chain-like molecules, e.g. polymers and alkanes, have been of intense theoretical and technological interest for the past few decades. Several accurate theories have been proposed to predict the properties of homonuclear chain-like fluids such as polymers and normal alkanes. ${ }^{1-5}$ Recent efforts, however, have focussed on the development of equations of state for heteronuclear chainlike fluids, e.g. copolymers. ${ }^{6-9}$ Copolymers offer chemists the opportunity to tailor new materials with desirable features because their properties can be manipulated not only by varying the chemical structure of the individual components but also by altering the arrangement of the monomeric species along the chain. For example, the arrangement of the monomeric species in a block copolymer molecule (diblock, triblock, or multiblock) strongly affects the type of microscopic phase segregation observed, and hence influences its mechanical properties. ${ }^{10}$ Despite the enormous technological importance of copolymer fluids and of blends containing copolymers, our understanding of the molecular basis for their thermophysical behavior is far from complete. Scientists and engineers attempting to predict the thermodynamic properties of copolymeric fluids (e.g. the miscibility of a copolymer-homopolymer blend) still rely on the Flory-Huggins ${ }^{11,12}$ theory, a theory developed almost half a century ago.

In this laboratory, we are conducting a theoretical study aimed at understanding the molecular underpinnings of the macroscopic thermodynamic behavior of copolymeric fluids. As a first step we recently developed new Generalized Flory (GF) and Generalized Flory-Dimer (GFD) equations of state which accurately predict the compressibility factor of copolymers modeled as hard heteronuclear chain fluids. ${ }^{8}$ How- ever, in order to develop a theory that will be suitable for practical applications we need to incorporate attractive interactions into our model. The simplest form of attractive interaction is the site-site square-well (SW) potential. In this paper we extend the Generalized Flory-Dimer formalism to copolymers modeled as heteronuclear chains whose segments interact via the site-site square-well potential. We obtain equations of states for three types of copolymer fluids: (1) block copolymers, (2) alternating copolymers, and (3) random copolymers. In order to test the accuracy of the GFD equation of state in predicting the compressibility factors of square-well copolymer fluids, we also perform extensive discontinuous canonical molecular dynamics (DCMD) simulations and compare the resulting compressibility factors to GFD theoretical predictions.

The approach used by Yethiraj and Hall ${ }^{13}$ to derive GFD equations of state for homonuclear square-well chains is extended to square-well heteronuclear chains. Using a combination of geometric and mean field arguments, Yethiraj and Hall showed that the GFD approach for homonuclear hard chains could be extended to homonuclear square-well chains by replacing the hard-monomer and hard-dimer insertion factors that appear in the homonuclear hard-chain insertion factor expression by square-well monomer and square-well dimer insertion factors. We use similar arguments to express the insertion factors of square-well block, alternating, and random copolymers in terms of the insertion factors for SW monomer mixtures, SW dimer mixtures, and SW heteronuclear dumbbell fluids obtained using second-order perturbation theory. ${ }^{14}$

Discontinuous canonical molecular dynamics simulation is an adaptation of Anderson's canonical ensemble molecular dynamics technique ${ }^{15}$ to the case in which the potential is 
discontinuous. The essential feature of Anderson's method is the introduction of stochastic energy fluctuations into an NVE ensemble such that the trajectory average of any property is equal to its NVT ensemble average. These stochastic energy fluctuations are introduced via periodic interaction of the system particles with imaginary constant-temperature heat bath particles. Using the DCMD technique, we have measured compressibility factors for square-well block, alternating, and random copolymers over a range of densities, temperatures, compositions, segment size ratios, and welldepth ratios.

A comparison of the GFD equation of state for squarewell copolymers with DCMD simulation results reveals that GFD accurately predicts the compressibility factor of squarewell block, alternating, and random copolymers, slightly overestimating it at very low densities and slightly underestimating it at very high densities. These trends are consistent with the trends observed in the GFD theory's predictions for homonuclear hard-chain and homonuclear square-well chain compressibility factors. We also study the effect of composition on the compressibility factor of block copolymers. The GFD theory is found to accurately predict the compressibility factor of block copolymers over the range of compositions studied.

The remainder of this paper is organized as follows: in Section II we briefly describe the molecular model and the computer simulation method used to study square-well heteronuclear chains. In Section III we review the GeneralizedFlory Dimer approaches for fluids containing homonuclear hard chains, homonuclear square-well chains, and heteronuclear hard chains. Section IV describes the Generalized Flory-Dimer theory for square-well block, alternating, and random copolymer fluids. In Section V we discuss the accuracy of the GFD theory in predicting the compressibility factors of square-well copolymer fluids by comparing theoretical predictions to DCMD simulation results.

\section{MOLECULAR MODEL AND SIMULATION METHOD}

In this section we describe the molecular model used and the simulation method employed to study copolymer fluids. Copolymer molecules are modeled as a pearl necklace chain of $n$ freely-jointed tangent square-well spheres of different hard-core sizes and/or different square-well depths. The site-site potential energy between segments $i$ and $j$, interacting via a square-well potential is given by

$$
\begin{aligned}
u_{i j}(r) & =\infty, \quad 0<r \leqslant \sigma_{i j}, \\
& =-\epsilon_{i j}, \quad \sigma_{i j}<r \leqslant \lambda_{i j} \sigma_{i j}, \\
& =0, \quad r>\lambda_{i j} \sigma_{i j},
\end{aligned}
$$

where $\sigma_{i j}=\left(\sigma_{i}+\sigma_{j}\right) / 2$ is the center-to-center distance between segments $i$ and $j, \lambda_{i j}$ is the square-well width, and $\epsilon_{i j}$ is the cross interaction energy between segments $i$ and $j$, taken to be

$$
\epsilon_{i j}=\sqrt{\epsilon_{i} \epsilon_{j}}
$$
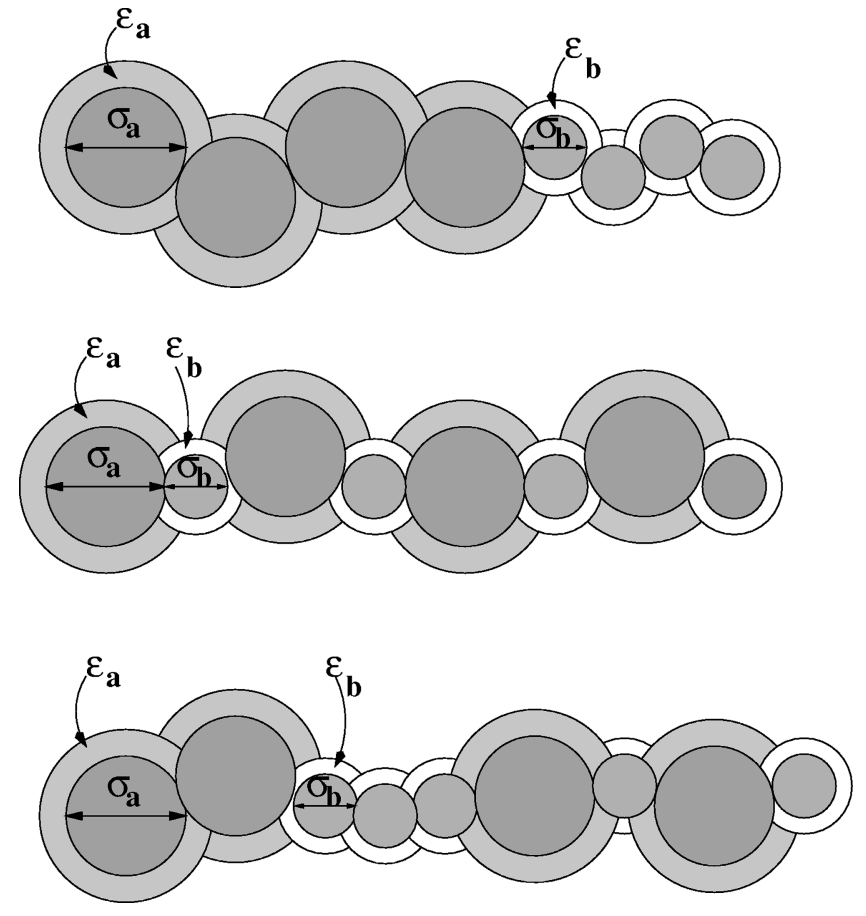

FIG. 1. Three types of heteronuclear square-well chain molecules studied: (a) block copolymer, (b) alternating copolymer, and (c) random copolymer.

In order to be consistent with previously available theoretical work on square-well fluids, we assume a square-well width of $\lambda_{i j}=\lambda=1.5$ throughout this work.

Figure 1 shows the three types of copolymer sequences: block, alternating and random, that are investigated in this paper. We restrict our analysis to copolymer chains composed of two types of segments, $a$ and $b$, although our formalism is equally applicable to heteronuclear fluids composed of more than two types of segments. The block copolymer chains consist of blocks of $n_{a}$ segments of component $a$ followed by $n_{b}$ segments of component $b$; the alternating copolymer chains consist of alternating segments of components $a$ and $b$, and the random copolymer chains consist of random sequences of components $a$ and component $b$. This simple "heteronuclear square-well chain" model qualitatively incorporates four of the most essential features of copolymer architecture: (1) steep repulsion between chains segments at short distances, (2) short-range attractions between segments, (3) connectivity of segments along a chain, and (4) chain topology or arrangement of various components along the chain.

In order to study these square-well chain models via computer simulation, we use the discontinuous molecular dynamics $^{16}$ (DMD) simulation technique. DMD on fluids containing tangent square-well spheres is implemented using the bead-string algorithm introduced by Rapaport ${ }^{17,18}$ and later modified by Bellemans. ${ }^{19}$ In the Rapaport algorithm, chain connectivity is maintained by linking adjacent spheres along a chain with short invisible strings. This effectively decouples the motion of bonded spheres along the chain, allowing them to move independently along linear trajectories between core collisions and bond stretches. Square-well interactions are accommodated in the DMD algorithm by 
introducing "well-capture," "well-bounce," and "welldissociation" collisions which occur whenever a sphere enters or leaves the square-well of any other sphere.

For square-well fluids in a constant temperature system, we use discontinuous canonical molecular dynamics (DCMD), ${ }^{14,15,20,21}$ an adaptation of the standard DMD technique for the canonical ensemble. The DCMD technique is based on Anderson's stochastic collision method ${ }^{15}$ and involves stochastic interaction of the system particles with imaginary constant-temperature heat bath particles. We assume that the system is immersed in an imaginary constanttemperature heat bath containing imaginary "ghost" particles. The ghost particles stabilize the system temperature by colliding with the system particles, as a result of which the particle velocities get reassigned according to a MaxwellBoltzmann distribution about the required temperature. Details of the discontinuous canonical molecular dynamics (DCMD) method have been described in previous publications. ${ }^{14,21}$

The DCMD simulations are performed at volume fractions $\eta$ ranging from 0.09 to 0.45 where $\eta$ $=\pi \Sigma N_{i} \sigma_{i}^{3} /(6 V)$ with $N_{i}$ equal to the number of segments of species $i$, and $V$ equal to the volume of the primary simulation cell. The compressibility factors are obtained for a wide range of block, alternating, and random copolymer fluids with diameter ratios, $\sigma_{b} / \sigma_{a}=1$ and 2 and well-depth ratios, $\epsilon_{b} / \epsilon_{a}$, ranging from 0.5 to 1.5 . The compressibility factor is calculated using an equation derived from the virial theorem of Clausius, ${ }^{22}$

$$
Z=\frac{N_{s}}{N_{t}}-\frac{1}{3 N_{t} k T t_{e}} \sum_{\text {colls }} m_{i} \mathbf{r}_{\mathbf{i j}} \cdot \Delta \mathbf{v}_{\mathbf{i}},
$$

where $N_{s}$ is the total number of segments in the system and $N_{t}$ is the total number of molecules in the system. Here $\mathbf{r}_{\mathbf{i j}}$ $=\mathbf{r}_{\mathbf{i}}-\mathbf{r}_{\mathbf{j}}$, where $\mathbf{r}_{\mathbf{i}}$ and $\mathbf{r}_{\mathbf{j}}$ are the position vectors of the colliding particles $i$ and $j$. The quantity $m_{i} \Delta \mathbf{v}_{\mathbf{i}}$ is the collisional impulse experienced by particle $i$ and is a measure of the force exerted by particle $j$ on particle $i$. The collisional virial is summed over all intermolecular and intramolecular core collisions, bond stretches, and square-well collisions occurring during the elapsed simulation time $t_{e}$. Since ghost collisions involve only one system particle, they do not contribute to the collisional virial.

\section{REVIEWING THE GENERALIZED FLORY-DIMER THEORY}

The basis for the Generalized Flory approaches is the so-called osmotic equation of state which relates the pressure of an $n$-mer chain fluid to the chain insertion factor, $p_{n}(\eta)$,

$$
\frac{P(\eta, n)}{k T}=\frac{\eta}{v_{n}}\left[1-\ln p_{n}(\eta)\right]+\frac{1}{v_{n}} \int_{0}^{\eta} \ln p_{n}\left(\eta^{\prime}\right) d \eta^{\prime},
$$

where $P(\eta, n)$, is the pressure of a fluid containing $n$-mers at volume fraction $\eta$ and temperature $T$, and $v_{n}$ is the volume of the chain. The insertion factor is defined as

$$
p_{n}(\eta)=\left\langle e^{-U / k T}\right\rangle
$$

where $U$ is the potential energy experienced by a test chain of length $n$ when inserted into a sea of chains (also of length $n)$. For hard chains, $\exp (-U / k T)$ takes on the values of 0 and 1 , leading to the interpretation of $p_{n}(\eta)$ as the chain insertion probability. The compressibility factor $Z$ $\equiv P(\eta, n) v_{n} / \eta k T$ can be expressed in terms of the chain insertion probability as

$$
Z=\left[1-\ln p_{n}(\eta)\right]+\frac{1}{\eta} \int_{0}^{\eta} \ln p_{n}\left(\eta^{\prime}\right) d \eta^{\prime},
$$

Since Eq. (6) is thermodynamically exact, the problem of obtaining an accurate equation of state for a given type of chain fluid reduces to the problem of developing an accurate estimate for its insertion factor. Given the complex nature of the structure of a chain fluid and the vast number of possible conformations that a chain may adopt, the chain insertion factor is a difficult quantity to calculate analytically. The Generalized Flory-Dimer ${ }^{23}$ theory provides a simple method for estimating the chain insertion factor in terms of the insertion factors for monomer and dimer fluids. Below, we briefly describe the GFD approach for hard homonuclear chains, hard heteronuclear chains, and square-well homonuclear chains.

\section{A. Generalized Flory Dimer theory for hard homonuclear chain fluids}

The GFD theory's estimate for the probability of inserting a hard homonuclear chain into a hard homonuclear chain fluid is

$$
p_{n}^{\mathrm{hs}}(\eta)=p_{1}^{\mathrm{hs}}(\eta)\left[\frac{p_{2}^{\mathrm{hs}}(\eta)}{p_{1}^{\mathrm{hs}}(\eta)}\right]^{\left[v_{e}(n)-v_{e}(1)\right] /\left[v_{e}(2)-v_{e}(1)\right]},
$$

where $p_{1}^{\mathrm{hs}}(\eta)$ is the probability of inserting a hard monomer into a hard $n$-mer fluid, $p_{2}^{\text {hs }}(\eta)$ is the probability of inserting a hard dimer into a hard $n$-mer fluid, and $v_{e}(n)$ is the volume excluded by an $n$-mer to a monomer. Equation (7) can be understood by imagining that the test chain is inserted into the chain fluid one segment at a time. The first term $p_{1}^{\text {hs }}(\eta)$ in Eq. (7) is the probability of inserting the first segment into the chain fluid. The second term in Eq. (7) is the product of the conditional probabilities,

$$
\frac{p_{k}^{\mathrm{hs}}(\eta)}{p_{k-1}^{\mathrm{hs}}(\eta)}=\left[\frac{p_{2}^{\mathrm{hs}}(\eta)}{p_{1}^{\mathrm{hs}}(\eta)}\right]^{\left[v_{e}(k)-v_{e}(k-1)\right] /\left[v_{e}(2)-v_{e}(1)\right]},
$$

of inserting the $k$ th segment $(k=2, \ldots, n)$ given that $k$ -1 segments have already been inserted, $p_{2}^{\mathrm{hs}}(\eta) / p_{1}^{\mathrm{hs}}(\eta)$ is the conditional probability of inserting the second bead next to the first bead, and the exponent in Eq. (8) corrects for the difference between the size of the hole required to place the $k$ th bead next to the $k-1$ st bead and the size of the hole required to place the second bead next to the first segment.

Using mean-field arguments, the probabilities, $p_{1}^{\mathrm{hs}}(\eta)$ and $p_{2}^{\mathrm{hs}}(\eta)$, of inserting monomers and dimers into a chain fluid are approximated by the corresponding probabilities of inserting a monomer into a monomer fluid and a dimer into a dimer fluid. When Eq. (7) is inserted into the osmotic equation of state, the resulting compressibility factor of a hard $n$-mer fluid is 


$$
Z_{n}^{\mathrm{hs}}(\eta)=\left(Y_{n}+1\right) Z_{2}^{\mathrm{hs}}(\eta)-Y_{n} Z_{1}^{\mathrm{hs}}(\eta),
$$

where $Z_{2}^{\mathrm{hs}}(\eta)$ and $Z_{1}^{\mathrm{hs}}(\eta)$ are the compressibility factors of hard dimer and hard monomer fluids, and

$$
Y_{n}=\frac{v_{e}(n)-v_{e}(2)}{v_{e}(2)-v_{e}(1)} .
$$

The monomer and dimer compressibility factors can be obtained from the Carnahan-Starling ${ }^{24}$ and Tildesley-Street ${ }^{25}$ equations of state, respectively. The excluded volume of an $n$-mer can be approximated by a linear function of chain length as ${ }^{26}$

$$
v_{e}(n) \approx v_{e}(3)+(n-3)\left[v_{e}(3)-v_{e}(2)\right],
$$

for $2 \leqslant n \leqslant 8$ and

$$
v_{e}(n) \approx v_{e}(1)[10.094+0.6374(n-15)],
$$

for $n>8 .^{27}$

\section{B. Generalized Flory-Dimer theory for homonuclear square-well chain fluids}

The Generalized Flory-Dimer theory has also been extended to fluids containing homonuclear square-well chains. ${ }^{5}$ Using a combination of geometric and mean field arguments, Yethiraj and $\mathrm{Hall}^{5}$ were able to show that the GFD factor for inserting a square-well chain into a square-well chain fluid, $p_{n}^{\mathrm{sw}}(\eta)$, can be expressed in terms of the factors for inserting a square-well monomer into a square-well monomer fluid, $p_{1}^{\mathrm{sw}}(\eta)$, and a square-well dimer into a square-well dimer fluid, $p_{2}^{\mathrm{sw}}(\eta)$

$$
p_{n}^{\mathrm{sw}}(\eta)=p_{1}^{\mathrm{sw}}(\eta)\left[\frac{p_{2}^{\mathrm{sw}}(\eta)}{p_{1}^{\mathrm{sw}}(\eta)}\right]^{\left[v_{e}(n)-v_{e}(1)\right] /\left[v_{e}(2)-v_{e}(1)\right]} .
$$

Thus we see that the Generalized Flory-Dimer approximation for the homonuclear hard chain insertion factor [Eq. (7)] is also applicable to homonuclear square-well chains if we replace the hard-monomer and hard dimer insertion factors by the square-well monomer and square-well dimer insertion factors.

By substituting the above estimate for the square-well chain insertion factor into the osmotic equation of state [Eq. (6)] we obtain the Generalized Flory-Dimer expression for the compressibility factor of a fluid containing square-well homonuclear chains,

$$
Z_{n}^{\mathrm{sw}}(\eta)=\left(Y_{n}+1\right) Z_{2}^{\mathrm{sw}}(\eta)-Y_{n} Z_{1}^{\mathrm{sw}}(\eta),
$$

where $Z_{2}^{\mathrm{sw}}(\eta)$ and $Z_{1}^{\mathrm{sw}}(\eta)$ are the compressibility factors of square-well dimer and square-well monomer fluids, and $Y_{n}$ is the excluded volume ratio as defined in Eq. (10). The square-well monomer ${ }^{28}$ and dimer ${ }^{13}$ compressibility factors can be obtained from perturbation theory or by using the RISM theory with mean-spherical approximation closure.

\section{Generalized Flory-Dimer theory for hard heteronuclear hard chains}

The GFD approach has recently been extended to hard heteronuclear chain fluids. ${ }^{8}$ The probability of inserting a copolymer chain into a copolymer fluid is again calculated by imagining that the test chain is inserted one segment at a time; the insertion probability of the chain is the product of the probabilities of inserting each of the segments. In this section we review the Generalized Flory-Dimer theories for block, alternating, and random hard copolymer fluids.

\section{Hard block copolymer chain fluids}

Consider an $n$-mer diblock copolymer fluid with each chain composed of $n_{a}$ segments of component $a$ followed by $n_{b}$ segments of component $b\left(n=n_{a}+n_{b}\right)$. The probability of inserting a test block copolymer chain into the block copolymer fluid is the product of the probabilities of inserting each of its blocks. The GFD arguments described in Sec. III A can be used to express the insertion probability of each block separately in terms of the probabilities of inserting monomers into monomer mixtures and dimers into dimer mixtures. The connectivity of the two blocks is incorporated by focusing on the two inner segments (the last segment on block $a$ and the first segment on block $b$ ) connecting the two blocks. The probability of inserting the first segment on block $b$ next to the last segment on block $a$ is approximated by the conditional probability of inserting the second segment of a heteronuclear dumbbell, given that the first segment has already been inserted. The resulting GFD expression for the probability of inserting a hard block copolymer chain into hard block copolymer fluid is

$$
\begin{aligned}
p_{n}^{\text {block }}(\eta)_{\mathrm{hs}}^{G F D}= & {\left[\frac{p_{2}^{a}(\eta)_{\mathrm{hs}}}{p_{1}^{a}(\eta)_{\mathrm{hs}}}\right]^{\left(Y_{n_{a}}^{a}+1\right)_{\text {mix }}} \times p_{2}^{a b}(\eta)_{\mathrm{hs}} } \\
& \times\left[\frac{p_{2}^{b}(\eta)_{\mathrm{hs}}}{p_{1}^{b}(\eta)_{\mathrm{hs}}}\right]^{\left(Y_{n_{b}}^{b}+1\right)_{m i x}},
\end{aligned}
$$

where $p_{1}^{a}(\eta)_{\mathrm{hs}}$ is the probability of inserting a hard-monomer of component $a$ into the hypothetical hard-monomer mixture formed by breaking all bonds of the block copolymer fluid, $p_{2}^{a}(\eta)_{\mathrm{hs}}$ is the probability of inserting a hard-dimer of component $a$ into the hypothetical hard-dimer mixture formed by breaking every other bond along the block copolymer chain, and $p_{2}^{a b}(\eta)_{\mathrm{hs}}$ is the probability of inserting a hardheteronuclear dumbbell into a hard heteronuclear dumbbell fluid.

The mixture excluded volume ratio, $\left(Y_{n_{a}}^{a}\right)_{m i x}$, is defined to be the occupation fraction average of the individual component excluded volume ratios,

$$
\left(Y_{n_{a}}^{a}\right)_{m i x}=\phi_{a}\left(Y_{n_{a}}^{a}\right)_{a}+\phi_{b}\left(Y_{n_{a}}^{a}\right)_{b},
$$

where $\phi_{a}$ is the occupation fraction, $\eta_{a} / \eta$, of component $a$ and $\left(Y_{n_{a}}^{a}\right)_{a}$ and $\left(Y_{n_{a}}^{a}\right)_{b}$ are calculated in a manner similar to Eq. (10),

$$
\left(Y_{n_{a}}^{a}\right)_{a}=\left(\frac{v_{e}^{a}\left(n_{a}\right)-v_{e}^{a}(2)}{v_{e}^{a}(2)-v_{e}^{a}(1)}\right)_{a}
$$

and 


$$
\left(Y_{n_{a}}^{a}\right)_{b}=\left(\frac{v_{e}^{a}\left(n_{a}\right)-v_{e}^{a}(2)}{v_{e}^{a}(2)-v_{e}^{a}(1)}\right)_{b}
$$

where $\left(v_{e}^{a}\left(n_{a}\right)\right)_{a}$ and $\left(v_{e}^{a}\left(n_{a}\right)\right)_{b}$ are the volumes excluded by a chain of $n_{a}$ segments of component $a$ to a monomer of component $a$ and to a monomer of component $b$, respectively. The expressions for excluded volumes $\left(v_{e}^{a}\left(n_{a}\right)\right)_{a}$ and $\left(v_{e}^{a}\left(n_{a}\right)\right)_{b}$ are presented in Appendix A.

Substituting Eq. (15) into the osmotic equation of state [Eq. (6)] we obtain the GFD expression for the compressibility factor of block copolymer hard-chain fluids,

$$
\begin{aligned}
Z_{n}^{\text {block }}(\eta)_{\mathrm{hs}}^{G F D}= & \left(Y_{n_{a}}^{a}+1\right)_{m i x}\left(\zeta_{2, \mathrm{hs}}^{a}-\zeta_{1, \mathrm{hs}}^{a}\right)+\zeta_{2, \mathrm{hs}}^{a b} \\
& +\left(Y_{n_{b}}^{b}+1\right)_{m i x}\left(\zeta_{2, \mathrm{hs}}^{b}-\zeta_{1, \mathrm{hs}}^{b}\right),
\end{aligned}
$$

where the $\zeta_{i \text {,hs }}^{a}$ are obtained by substituting the $p_{i}^{a}(\eta)_{\mathrm{hs}}$ (probabilities of inserting an $i$-mer of species $a$ into an $i$-mer mixture) into the osmotic equation of state,

$$
\zeta_{i, \mathrm{hs}}^{a}=\left[1-\ln p_{i}^{a}(\eta)_{\mathrm{hs}}\right]+\frac{1}{\eta} \int_{0}^{\eta} \ln p_{i}^{a}\left(\eta^{\prime}\right)_{\mathrm{hs}} d \eta^{\prime}
$$

with similar expressions for $\zeta_{i \text {, hs }}^{b}$ and $\zeta_{2, \mathrm{hs}}^{a b}$. The expressions for $\zeta_{i \text {, hs }}$ for monomers, dimers and heteronuclear dumbbells can be obtained using the scaled particle theory ${ }^{29,30}$ and are shown in Appendix B.

\section{Hard alternating copolymer chain fluids}

We now consider a hard chain composed of alternating segments of component $a$ and $b$. The Generalized FloryDimer estimate for the probability of inserting an alternating copolymer of $n$ segments into an alternating copolymer fluid is obtained using a combination of conditional probability and mean field arguments. The probability of inserting the alternating copolymer is again taken to be the product of the insertion probabilities of each of its segments. The probability of inserting each segment is calculated in terms of the conditional probability of inserting the second segment of an alternating dumbbell next to the first and an excluded volume argument. The resulting GFD expression for the insertion probability of an alternating copolymer is given by

$p_{n}^{\text {alter }}(\eta)_{\mathrm{hs}}^{G F D}=p_{2}^{a b}(\eta)_{\mathrm{hs}}\left[\frac{p_{2}^{b a}(\eta)_{\mathrm{hs}}}{p_{1}^{b}(\eta)_{\mathrm{hs}}}\right]^{\left(W_{n}^{a}\right)_{m i x}}\left[\frac{p_{2}^{a b}(\eta)_{\mathrm{hs}}}{p_{1}^{a}(\eta)_{\mathrm{hs}}}\right]^{\left(W_{n}^{b}\right)_{\text {mix }}}$,

where the mixture excluded volume ratios $\left(W_{n}^{a}\right)_{\text {mix }}$ and $\left(W_{n}^{b}\right)_{\text {mix }}$ are defined as the occupation fraction averages,

$$
\left(W_{n}^{a}\right)_{m i x}=\phi_{a}\left(W_{n}^{a}\right)_{a}+\phi_{b}\left(W_{n}^{a}\right)_{b},
$$

with a similar expression for $\left(W_{n}^{b}\right)_{\text {mix }}$. The individual component excluded volume arguments, $W_{n}$ 's, are defined as

$$
\left(W_{n}^{i}\right)_{k}=\left(\frac{n}{2}-1\right)\left(\frac{v_{e}^{i j i}(3)-v_{e}^{i j}(2)}{v_{e}^{j i}(2)-v_{e}^{j}(1)}\right)_{\left.k\right|_{i \neq j}},
$$

where $\left(v_{e}^{i j i}(3)\right)_{k}$ and $\left(v_{e}^{j i}(2)\right)_{k}$ are the volumes excluded by an $i j i$ alternating trimer and a $j i$ dumbbell to a segment of component $k$. The expressions for these excluded volumes are given in Appendix A.

The resulting GFD expression for the compressibility factor of an alternating copolymer chain fluid is

$$
\begin{aligned}
Z_{n}^{\text {alter }}(\eta)_{\mathrm{hs}}^{G F D}= & \zeta_{2, \mathrm{hs}}^{a b}+\left(W_{n}^{a}\right)_{m i x}\left(\zeta_{2, \mathrm{hs}}^{a b}-\zeta_{1, \mathrm{hs}}^{b}\right) \\
& +\left(W_{n}^{b}\right)_{m i x}\left(\zeta_{2, \mathrm{hs}}^{a b}-\zeta_{1, \mathrm{hs}}^{a}\right),
\end{aligned}
$$

where $\zeta_{2, \mathrm{hs}}^{a b}$ is obtained by substituting $p_{2}^{a b}(\eta)_{\mathrm{hs}}$ (the probability for inserting an $a b$ dumbbell into a dumbbell fluid) into the osmotic equation of state [Eq. (6)]. The analytical expressions for $\zeta_{1, \mathrm{hs}}^{i}$ and $\zeta_{2, \mathrm{hs}}^{a b}$ are given in Appendix B.

\section{Hard random copolymer chain fluids}

The extension of the Generalized Flory-Dimer theory to random copolymer chain fluids requires a combination of the block and alternating copolymer GFD approaches described earlier and mathematical probability arguments to approximate the average structure of the random copolymer. The GFD expression for the insertion probability of a random copolymer is

$$
\begin{aligned}
& p_{n}^{\text {rand }}(\eta)_{\mathrm{hs}}^{G F D}=\left(p_{1}^{a}(\eta)_{\mathrm{hs}}\right)^{x_{a}}\left(p_{1}^{b}(\eta)_{\mathrm{hs}}\right)^{x_{b}}\left(\frac{p_{2}^{a}(\eta)_{\mathrm{hs}}}{p_{1}^{a}(\eta)_{\mathrm{hs}}}\right)^{\left(\mathscr{U}_{N_{a a}}^{a}\right)_{m i x}} \\
& \times\left(\frac{p_{2}^{b}(\eta)_{\mathrm{hs}}}{p_{1}^{b}(\eta)_{\mathrm{hs}}}\right)^{\left(\mathscr{U}_{N_{b b}}^{b}\right)_{\text {mix }}}\left(\frac{p_{2}^{a b}(\eta)_{\mathrm{hs}}}{p_{1}^{a}(\eta)_{\mathrm{hs}}}\right)^{\left(\mathscr{H}_{N_{a b}}^{b}\right)_{\text {mix }}} \\
& \times\left(\frac{p_{2}^{b a}(\eta)_{\mathrm{hs}}}{p_{1}^{b}(\eta)_{\mathrm{hs}}}\right)^{\left(\mathscr{\mathscr { H }}_{N_{b a}}^{a}\right)_{m i x}}
\end{aligned}
$$

where $x_{i}$ is the mole fraction of component $i$. The exponents $\left(\mathscr{W}_{N_{j i}}^{i}\right)_{\text {mix }}$ are defined as

$$
\left(\mathscr{W}_{N_{j i}}^{i}\right)_{m i x}=(n-1) x_{i} x_{j}\left(\frac{\bar{v}_{e}^{l j i}(3)-\bar{v}_{e}^{l j}(2)}{v_{e}^{j i}(2)-v_{e}^{j}(1)}\right)_{\text {mix }},
$$

where the factor $(n-1) x_{i} x_{j}$ accounts for the average number of $i j$ pairs in a random copolymer chain. The excluded volume ratio in Eq. (26) is defined as

$$
\begin{aligned}
\left(\frac{\bar{v}_{e}^{l j i}(3)-\bar{v}_{e}^{l j}(2)}{v_{e}^{j i}(2)-v_{e}^{j}(1)}\right)_{m i x}= & \phi_{a}\left(\frac{\bar{v}_{e}^{l j i}(3)-\bar{v}_{e}^{l j}(2)}{v_{e}^{j i}(2)-v_{e}^{j}(1)}\right)_{a} \\
& +\phi_{b}\left(\frac{\bar{v}_{e}^{l j i}(3)-\bar{v}_{e}^{l j}(2)}{v_{e}^{j i}(2)-v_{e}^{j}(1)}\right)_{b},
\end{aligned}
$$

where the term $\left(\bar{v}_{e}^{l j i}(3)-\bar{v}_{e}^{l j}(2)\right)_{a}$ is the volume required to insert a segment of component $i$ next to a dimer of the form $l j$ averaged over all possible values of $l$ (consistent with the random copolymer's composition) in a fluid containing monomers of type $a$ (as indicated by the subscript),

$$
\begin{aligned}
\left(\bar{v}_{e}^{l j i}(3)-\bar{v}_{e}^{l j}(2)\right)_{a}= & x_{a}\left(v_{e}^{a j i}(3)-v_{e}^{a j}(2)\right)_{a} \\
& +x_{b}\left(v_{e}^{b j i}(3)-v_{e}^{b j}(2)\right)_{a} .
\end{aligned}
$$


The exponents $\left(\mathscr{\mathscr { V }}_{N_{j i}}^{i}\right)_{\text {mix }}$ require the expressions for excluded volumes of trimers $a a b, b a b, a b a$, and $b b a$. These are presented in Appendix A.

The compressibility factor is again calculated by substituting the insertion probability expression into the osmotic equation of state to obtain

$$
\begin{aligned}
Z_{n}^{\text {rand }}(\eta)_{\mathrm{hs}}^{G F D}= & x_{a} \zeta_{1, \mathrm{hs}}^{a}+x_{b} \zeta_{1, \mathrm{hs}}^{b}+\left(\mathscr{\mathscr { W }}_{N_{a a}}^{a}\right)_{m i x}\left(\zeta_{2, \mathrm{hs}}^{a}-\zeta_{1, \mathrm{hs}}^{a}\right) \\
& +\left(\mathscr{W}_{N_{b b}}^{b}\right)_{m i x}\left(\zeta_{2, \mathrm{hs}}^{b}-\zeta_{1, \mathrm{hs}}^{b}\right) \\
& +\left(\mathscr{\mathscr { W }}_{N_{a b}}^{b}\right)_{m i x}\left(\zeta_{2, \mathrm{hs}}^{a b}-\zeta_{1, \mathrm{hs}}^{a}\right) \\
& +\left(\mathscr{W}_{N_{b a}}^{a}\right)_{m i x}\left(\zeta_{2, \mathrm{hs}}^{b a}-\zeta_{1, \mathrm{hs}}^{b}\right) .
\end{aligned}
$$

The analytical expressions for $\zeta_{1, \text { hs }}^{i}$ and $\zeta_{2, \mathrm{hs}}^{i}$ are shown in Appendix B.

\section{EXTENSION OF GENERALIZED FLORY-DIMER THEORY TO SQUARE-WELL COPOLYMERS}

In this section we describe the extension of the Generalized Flory-Dimer theory to heteronuclear square-well chain molecules. We use the approach introduced by Yethiraj and Hall $^{13}$ to extend the GFD theory for homonuclear hard chains to homonuclear square-well chains. As stated in Sec. III B, Yethiraj and Hall have shown that the GFD approximation for the homonuclear hard chain insertion factor is applicable to square-well chains if we replace the hardmonomer and hard-dimer insertion factors by the square-well monomer and square-well dimer insertion factors. Following the same arguments, the GFD approximation for the heteronuclear hard-chain insertion factor is applicable to the heteronuclear square-well chain insertion factor if we replace the hard-monomer mixture, hard-dimer mixture, and hardheteronuclear dumbbell insertion factors by the corresponding square-well monomer-mixture, square-well dimer mixture, and square-well heteronuclear dumbbell insertion factors. Below we show the expressions for compressibility factors of square-well copolymers resulting from the above substitution.

\section{A. Square-well block copolymers}

The insertion factor for a square-well block copolymer chain can be obtained from Eq. (15) by replacing these hardmonomer mixture, hard-dimer mixture, and hardheteronuclear dumbbell insertion probabilities by the corresponding square-well monomer mixture, square-well dimer mixture, and square-well heteronuclear dumbbell insertion factors. The resulting expression for the insertion factor of a square-well block copolymer chain in a square-well block copolymer fluid is

$$
\begin{aligned}
p_{n}^{\text {block }}(\eta)_{\mathrm{sw}}^{\mathrm{GFD}} & \\
= & {\left[\frac{p_{2}^{a}(\eta)_{\mathrm{sw}}}{p_{1}^{a}(\eta)_{\mathrm{sw}}}\right]^{\left(Y_{n_{a}}^{a}+1\right)_{\text {mix }}} p_{2}^{a b}(\eta)_{\mathrm{sw}}\left[\frac{p_{2}^{b}(\eta)_{\mathrm{sw}}}{p_{2}^{b}(\eta)_{\mathrm{sw}}}\right]^{\left(Y_{n_{b}}^{b}+1\right)_{m i x}}, }
\end{aligned}
$$

where $p_{1}^{i}(\eta)_{\mathrm{sw}}$ is the insertion factor for a SW monomer of species $i$ in the hypothetical SW monomer mixture formed by breaking all of the bonds of the square-well block copolymer fluid, $p_{2}^{i}(\eta)_{\mathrm{sw}}$ is the insertion factor for a SW dimer of species $i$ in the hypothetical SW dimer mixture formed by breaking every other bond along the block copolymer chains, and $p_{2}^{a b}(\eta)_{\mathrm{sw}}$ is the insertion factor for a square-well heteronuclear dumbbell in a square-well heteronuclear dumbbell fluid at the same density as the copolymer fluid. The excluded volume arguments $\left(Y_{n_{a}}^{a}\right)_{\text {mix }}$ and $\left(Y_{n_{b}}^{b}\right)_{\text {mix }}$ are the same excluded volume arguments used to determine the insertion factor for hard block copolymer chains and can be calculated as shown in Eqs. (16), (17), and (18).

The GFD expression for the compressibility factor of a square-well block copolymer fluid is obtained by substituting Eq. (30) into the osmotic equation of state [Eq. (6)] to obtain

$$
\begin{aligned}
Z_{n}^{\text {block }}(\eta)_{\mathrm{sw}}^{\mathrm{GFD}}= & \left(Y_{n_{a}}^{a}+1\right)_{m i x}\left(\zeta_{2, \mathrm{sw}}^{a}-\zeta_{1, \mathrm{sw}}^{a}\right) \\
& +\zeta_{2, \mathrm{sw}}^{a b}+\left(Y_{n_{b}}^{b}+1\right)_{m i x}\left(\zeta_{2, \mathrm{sw}}^{b}-\zeta_{1, \mathrm{sw}}^{b}\right),
\end{aligned}
$$

where the $\zeta_{i}^{a}$,s are defined as

$$
\zeta_{i, \mathrm{sw}}^{a}=\left[1-\ln p_{i}^{a}(\eta)_{\mathrm{sw}}\right]+\frac{1}{\eta} \int_{0}^{\eta} \ln p_{i}^{a}\left(\eta^{\prime}\right)_{\mathrm{sw}} d \eta^{\prime},
$$

with a similar expression for $\zeta_{i, \mathrm{sw}}^{b}$ and $\zeta_{2, \mathrm{sw}}^{a b}$. The expressions for $\zeta_{i, \mathrm{sw}}$ for monomers, dimers, and heteronuclear dumbbells can be obtained from second-order perturbation theory ${ }^{14}$ as shown in Appendix B.

\section{B. Square-well alternating copolymers}

The insertion factor for a square-well alternating copolymer chain can be obtained from Eq. (21) by replacing the hard-monomer mixture and hard-heteronuclear dumbbell insertion factors by the corresponding square-well monomer mixture and square-well heteronuclear dumbbell insertion factors. The resulting expression for the insertion factor of a square-well alternating copolymer chain in a square-well alternating copolymer fluid is

$$
\begin{aligned}
& p_{n}^{\text {alter }}(\eta)_{\mathrm{sw}}^{G F D} \\
& \quad=p_{2}^{a b}(\eta)_{\mathrm{sw}}\left[\frac{p_{2}^{b a}(\eta)_{\mathrm{sw}}}{p_{1}^{b}(\eta)_{\mathrm{sw}}}\right]^{\left(W_{n}^{a}\right)_{m i x}}\left[\frac{p_{2}^{a b}(\eta)_{\mathrm{sw}}}{p_{1}^{a}(\eta)_{\mathrm{sw}}}\right]^{\left(W_{n}^{b}\right)_{m i x}},
\end{aligned}
$$

where $p_{1}^{i}(\eta)_{\mathrm{sw}}$ is the insertion factor for a SW monomer of species $i$ in the hypothetical SW monomer mixture formed by breaking all of the bonds of the square-well alternating copolymer fluid and $p_{2}^{a b}(\eta)_{\mathrm{sw}}$ is the insertion factor for a square-well heteronuclear dumbbell in a square-well heteronuclear dumbbell fluid at the same density as the copolymer fluid. The excluded volume arguments $\left(W_{n}^{a}\right)_{\text {mix }}$ and $\left(W_{n}^{b}\right)_{\text {mix }}$ are the same excluded volume arguments used to determine the hard alternating copolymer insertion factor and can be calculated as shown in Eqs. (22) and (23). The GFD expression for the compressibility factor of a square-well alternating copolymer fluid is obtained by substituting Eq. (33) into the osmotic equation of state [Eq. (6)] to obtain

$$
\begin{aligned}
Z_{n}^{\text {alter }}(\eta)_{\mathrm{sw}}^{G F D}= & \zeta_{2, \mathrm{sw}}^{a b}+\left(W_{n}^{a}\right)_{m i x}\left(\zeta_{2, \mathrm{sw}}^{b a}-\zeta_{1, \mathrm{sw}}^{b}\right) \\
& +\left(W_{n}^{b}\right)_{m i x}\left(\zeta_{2, \mathrm{sw}}^{a b}-\zeta_{1, \mathrm{sw}}^{a}\right) .
\end{aligned}
$$


The expressions for $\zeta_{i, \mathrm{sw}}$ for monomers and heteronuclear dumbbells can be obtained using second-order perturbation theory ${ }^{14}$ as are shown in Appendix B.

\section{Square-well random copolymers}

The insertion factor for square-well random copolymer chains can be obtained from Eq. (25) by replacing the hardmonomer mixture, hard-dimer mixture, and hardheteronuclear dumbbell insertion factors by the corresponding square-well monomer mixture, square-well dimer mixture, and square-well heteronuclear dumbbell insertion factors. The resulting expression for the insertion factor of a square-well random copolymer chain in a square-well random copolymer fluid is

$$
\begin{aligned}
p_{n}^{\text {rand }(\eta)_{\mathrm{sw}}^{G F D}=} & \left(p_{1}^{a}(\eta)_{\mathrm{sw}}\right)^{x}\left(p_{1}^{b}(\eta)_{\mathrm{sw}}\right)^{x_{b}}\left(\frac{p_{2}^{a}(\eta)_{\mathrm{sw}}}{p_{1}^{a}(\eta)_{\mathrm{sw}}}\right)^{\left(\mathscr{U}_{N_{a a}}^{a}\right)_{m i x}} \\
& \times\left(\frac{p_{2}^{b}(\eta)_{\mathrm{sw}}}{p_{1}^{b}(\eta)_{\mathrm{sw}}}\right){ }^{\left(\mathscr{U}_{N_{b b}}^{b}\right)_{m i x}}\left(\frac{p_{2}^{a b}(\eta)_{\mathrm{sw}}}{p_{1}^{a}(\eta)_{\mathrm{sw}}}\right)^{\left(\mathscr{H}_{N_{a b}}^{b}\right)_{m i x}} \\
& \times\left(\frac{p_{2}^{b a}(\eta)_{\mathrm{sw}}}{p_{1}^{b}(\eta)_{\mathrm{sw}}}\right)^{\left(\mathscr{U}_{N_{b a}}^{a}\right)_{m i x}} .
\end{aligned}
$$

The excluded volume arguments $\left(\mathscr{\mathscr { W }}_{N_{a a}}^{a}\right)_{\text {mix }},\left(\mathscr{W}_{N_{b b}}^{b}\right)_{\text {mix }}$, $\left(\mathscr{W}_{N_{a b}}^{b}\right)_{\text {mix }}$, and $\left(\mathscr{W}_{N_{b a}}^{a}\right)_{\text {mix }}$ are the same as the excluded volume arguments used to determine the hard random copolymer insertion factor and can be calculated as shown in Eq. (26). The GFD expression for the compressibility factor of a square-well random copolymer fluid is obtained by substituting Eq. (35) into the osmotic equation of state [Eq. (6)] to obtain

$$
\begin{aligned}
Z_{n}^{r a n d}(\eta)_{\mathrm{sw}}^{G F D}= & x_{a} \zeta_{1, \mathrm{sw}}^{a}+x_{b} \zeta_{1, \mathrm{sw}}^{b}+\left(\mathscr{\mathscr { W }}_{N_{a a}}^{a}\right)_{m i x}\left(\zeta_{2, \mathrm{sw}}^{a}\right. \\
& \left.-\zeta_{1, \mathrm{sw}}^{a}\right)+\left(\mathscr{\mathscr { W }}_{N_{b b}}^{b}\right)_{m i x}\left(\zeta_{2, \mathrm{sw}}^{b}-\zeta_{1, \mathrm{sw}}^{b}\right) \\
& +\left(\mathscr{\mathscr { W }}_{N_{a b}}^{b}\right)_{m i x}\left(\zeta_{2, \mathrm{sw}}^{a b}-\zeta_{1, \mathrm{sw}}^{a}\right) \\
& +\left(\mathscr{W}_{N_{b a}}^{a}\right)_{m i x}\left(\zeta_{2, \mathrm{sw}}^{b a}-\zeta_{1, \mathrm{sw}}^{b}\right) .
\end{aligned}
$$

The expressions for $\zeta_{1, \mathrm{sw}}^{i}$ and $\zeta_{2, \mathrm{sw}}^{i}$ are shown in Appendix B.

\section{COMPARISONS OF GENERALIZED FLORY DIMER THEORY TO MOLECULAR DYNAMICS SIMULATION RESULTS}

In this section we compare the GFD predictions for the compressibility factors of square-well copolymers with the results of our DCMD simulations. We report compressibility factor for square-well block, alternating, and random copolymer fluids for a range of densities, compositions, segment size ratios, and square-well depth ratios. The duration of the simulation runs ranges from 10 million collisions for short chain lengths at low densities to 30 million collisions for longer chain lengths at high densities. The error bars on the compressibility factors calculated in these simulations represent \pm standard deviations as determined from three or more independent runs.

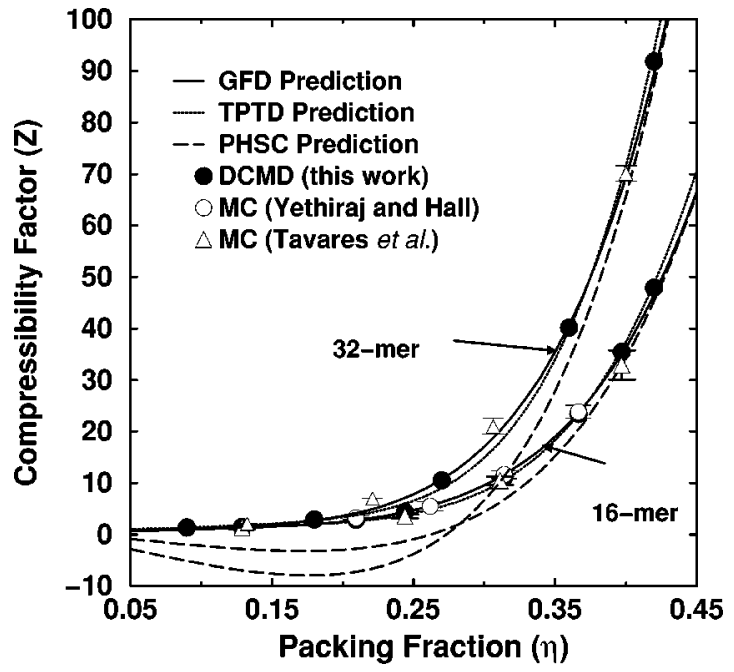

FIG. 2. Comparison of the predictions of GFD theory for homonuclear square-well chains with the results of our DCMD simulation and previous MC simulation by Yethiraj and $\mathrm{Hall}^{5}$ and Tavares et al. ${ }^{31}$ Results are shown for 16-mer and 32-mer fluids at $T^{*}=3$.

In order to test our DCMD simulation method, we begin by comparing the DCMD compressibility factors for homonuclear square-well chains with previously available MC data and with the predictions of various theories for homonuclear square-well chain fluids. Figure 2 shows the compressibility factor of 16-mer and 32-mer homonuclear square-well chains at $T^{*}=3$ as a function of packing fraction. The open circles represent the results of MC simulation by Yethiraj and Hall, ${ }^{5}$ the open triangles are the results of MC simulation by Tavares et al., ${ }^{31}$ and the filled circles are our DCMD simulation results. The solid lines in Fig. 2 are the predictions of the GFD theory by Yethiraj and Hall, ${ }^{5}$ dotted lines are the predictions of the TPT-D theory by Tavares et al. ${ }^{31}$ and the dashed lines are the predictions of PHSCT theory by Hino and Prausnitz. ${ }^{32}$ The MC simulation results of Yethiraj et al. and Tavares et al. are in excellent agreement with our DCMD simulation results for both chain lengths over the entire range of densities studied. The TPT-D theory of Tavares et al. and the GFD theory of Yethiraj and Hall accurately predict the compressibility factors for both chain lengths and the entire range of densities studied. The PHSCT theory of Hino and Prausnitz underpredicts the compressibility factor, especially at low densities.

\section{A. Square-well block copolymers}

Having established the accuracy of our discontinuous canonical molecular dynamics technique in predicting the compressibility factors of fluids composed of homonuclear molecules, we compare the predictions of GFD theory for square-well block copolymer fluids with the DCMD simulation results. Table I summarizes the simulation results and GFD predictions for all square-well block copolymer fluids considered. Figures 3-5 compare the GFD predictions with DCMD simulation results for the compressibility factors as a function of $\eta$ for square-well block copolymers composed of chains of length $n=4,8$, and 16 at $T^{*}=3.0$ and composition $x_{a}=0.5$. In Fig. 3 the two segments of the two components 
TABLE I. DCMD simulation values of compressibility factors for square-well block copolymers. The value in parentheses represents one standard deviation in the last two significant digits.

\begin{tabular}{|c|c|c|c|c|c|c|c|c|c|c|}
\hline \multirow[b]{2}{*}{$T^{*}$} & \multirow{2}{*}{$\frac{\sigma_{b}}{\sigma_{a}}$} & \multirow[b]{2}{*}{$\frac{\epsilon_{b}}{\epsilon_{a}}$} & \multirow[b]{2}{*}{$x_{a}$} & \multirow[b]{2}{*}{$\eta$} & \multicolumn{2}{|c|}{ 4-mer } & \multicolumn{2}{|c|}{ 8-mer } & \multicolumn{2}{|c|}{ 16-mer } \\
\hline & & & & & $\mathrm{Z}(\mathrm{MD})$ & $\mathrm{Z}(\mathrm{GFD})$ & $\mathrm{Z}(\mathrm{MD})$ & $\mathrm{Z}(\mathrm{GFD})$ & $\mathrm{Z}(\mathrm{MD})$ & $\mathrm{Z}(\mathrm{GFD})$ \\
\hline \multirow[t]{5}{*}{3} & 1 & 0.5 & 0.5 & 0.09 & $1.348(02)$ & 1.349 & $1.536(09)$ & 1.556 & $1.908(28)$ & 1.970 \\
\hline & & & & 0.18 & 2.137(04) & 2.006 & $2.852(14)$ & 2.575 & $4.190(32)$ & 3.715 \\
\hline & & & & 0.27 & $4.092(08)$ & 3.781 & $6.275(18)$ & 5.721 & $10.616(36)$ & 9.603 \\
\hline & & & & 0.36 & $9.282(11)$ & 8.541 & $15.844(22)$ & 14.841 & $28.851(76)$ & 27.441 \\
\hline & & & & 0.45 & $22.216(22)$ & 20.097 & $40.762(38)$ & 37.777 & $77.566(96)$ & 73.137 \\
\hline \multirow[t]{5}{*}{3} & 1 & 1.5 & 0.5 & 0.09 & $0.825(03)$ & 0.897 & $0.687(07)$ & 0.687 & $0.489(42)$ & 0.268 \\
\hline & & & & 0.18 & $0.958(03)$ & 0.787 & $0.816(16)$ & 0.095 & $0.510(29)$ & -1.288 \\
\hline & & & & 0.27 & $1.866(11)$ & 1.570 & $2.120(26)$ & 1.102 & $2.563(38)$ & 0.166 \\
\hline & & & & 0.36 & $5.760(09)$ & 5.449 & $8.869(28)$ & 8.258 & $14.972(10)$ & 13.876 \\
\hline & & & & 0.45 & $18.455(18)$ & 16.749 & $33.011(04)$ & 30.446 & $62.169(99)$ & 57.840 \\
\hline \multirow[t]{5}{*}{3} & 2 & 1 & 0.5 & 0.09 & $1.010(06)$ & 1.073 & $1.006(21)$ & 1.016 & $1.003(54)$ & 0.903 \\
\hline & & & & 0.18 & $1.291(11)$ & 1.212 & $1.367(24)$ & 0.974 & $1.489(40)$ & 0.500 \\
\hline & & & & 0.27 & $2.269(11)$ & 2.105 & $2.888(10)$ & 2.302 & $4.022(93)$ & 2.154 \\
\hline & & & & 0.36 & $5.649(17)$ & 5.399 & $8.750(39)$ & 8.411 & $14.814(85)$ & 14.435 \\
\hline & & & & 0.45 & $15.274(18)$ & 14.332 & $26.896(73)$ & 25.992 & $50.031(56)$ & 49.302 \\
\hline \multirow[t]{5}{*}{3} & 2 & 0.5 & 0.5 & 0.09 & $1.370(04)$ & 1.363 & $1.563(04)$ & 1.576 & $1.961(18)$ & 2.000 \\
\hline & & & & 0.18 & $2.125(03)$ & 1.997 & $2.787(10)$ & 2.577 & $4.081(27)$ & 3.735 \\
\hline & & & & 0.27 & $3.845(15)$ & 3.529 & $5.725(23)$ & 5.290 & 9.499(39) & 8.810 \\
\hline & & & & 0.36 & $7.997(16)$ & 7.389 & $13.300(32)$ & 12.669 & 23.994(79) & 23.224 \\
\hline & & & & 0.45 & $17.847(15)$ & 16.487 & $32.093(26)$ & 30.732 & $60.519(91)$ & 59.210 \\
\hline
\end{tabular}

of the block copolymer are of the same size $\left(\sigma_{b} / \sigma_{a}=1\right)$ while the relative well-depth is $\epsilon_{b} / \epsilon_{a}=0.5$. In Fig. 4 $\sigma_{b} / \sigma_{a}=2$, while the well-depths of the two components are equal $\left(\epsilon_{b} / \epsilon_{a}=1.0\right)$. In Fig. 5 the hard-core sizes and the well-depths of the two components are both different: $\sigma_{b} / \sigma_{a}=2, \epsilon_{b} / \epsilon_{a}=0.5$. As is evident from the figures, the GFD theory accurately predicts the compressibility factors of square-well block copolymers except at very low and very high densities.

The GFD theory is found to slightly overestimate the compressibility factor of square-well block copolymers at low densities and slightly underestimate the compressibility

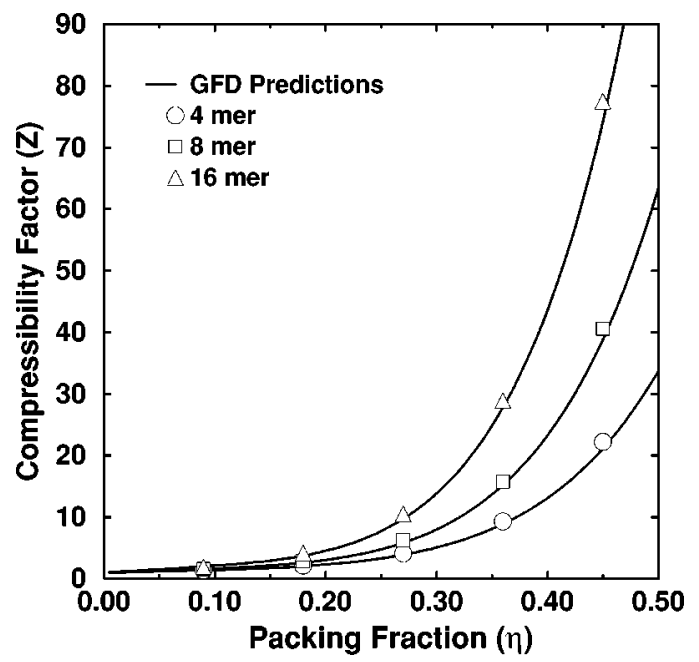

FIG. 3. Square-well block-copolymer compressibility factor vs packing fraction for 4-mers, 8-mers, and 16-mers at $T^{*}=3$ where $\sigma_{b} / \sigma_{a}=1.0, x_{a}$ $=0.5$, and $\epsilon_{b} / \epsilon_{a}=0.5$. factor at high densities. These trends are consistent with the trends observed in the GFD predictions for homonuclear and heteronuclear hard chains and are inherent deficiencies in the GFD approach. The error at low densities arises from the use of the mean field approximation which assumes that the structure of a copolymer fluid is essentially the same as the structure of the reference monomer and dimer mixtures. While this approximation works well at intermediate to high densities, the approximation is less successful at very low densities where the structure of the chain fluid is relatively patchy. We also observe in Fig. 4 that GFD slightly underpredicts the compressibility factor in the low to intermediate

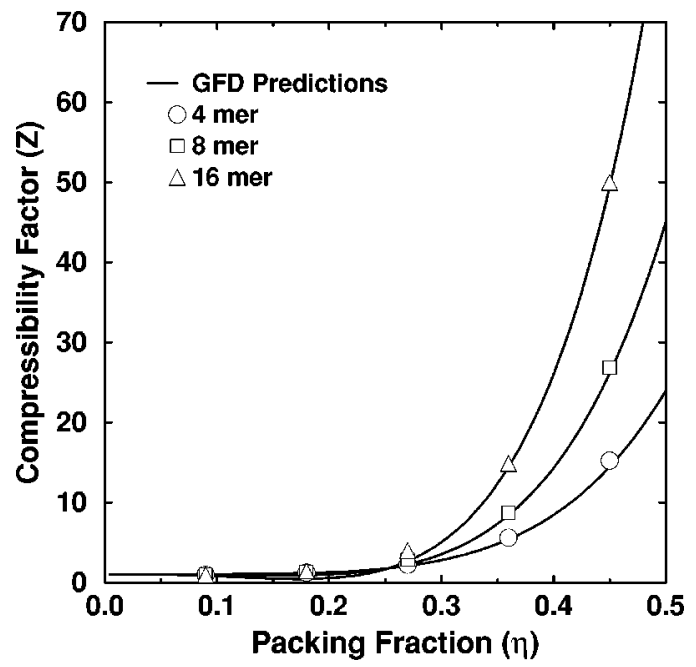

FIG. 4. Square-well block-copolymer compressibility factor vs packing fraction for 4-mers, 8-mers, and 16-mers at $T^{*}=3.0$ where $\sigma_{b} / \sigma_{a}=2.0$, $x_{a}=0.5$, and $\epsilon_{b} / \epsilon_{a}=1.0$. 


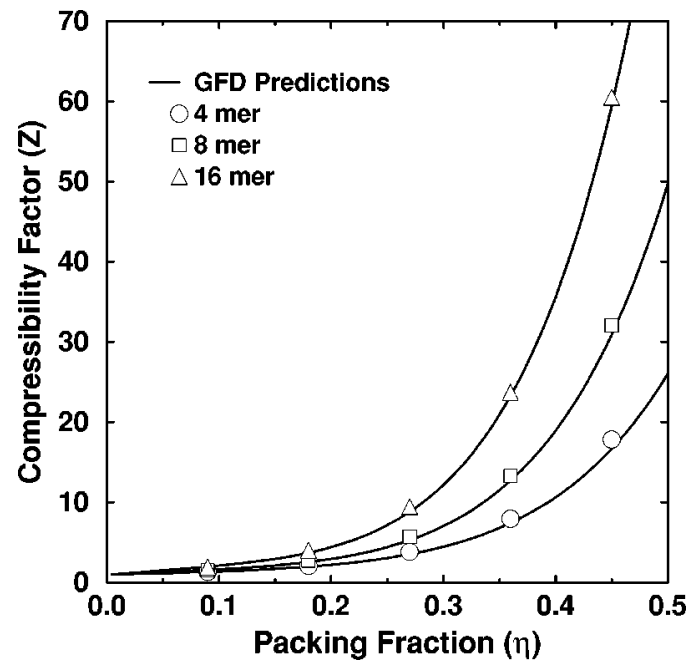

FIG. 5. Square-well block-copolymer compressibility factor vs packing fraction for 4-mers, 8-mers, and 16-mers at $T^{*}=3.0$ where $\sigma_{b} / \sigma_{a}=2.0$, $x_{a}=0.5$, and $\epsilon_{b} / \epsilon_{a}=0.5$.

density region. The perturbation theory used to calculate the insertion factors of square-well monomer mixtures and square-well dimer mixtures is not very accurate at these densities since these states may be very close to or in the two phase region. Additionally, the simulation data in these cases is also suspect as the periodic boundaries and the DCMD simulation technique used do not allow for phase separation.

Next we study how the compressibility factor of squarewell block copolymers varies with temperature and composition. Figure 6 shows the compressibility factor of a 16-mer square-well block copolymer fluid at $T^{*}=1.5,2$, and 3 where $\sigma_{b} / \sigma_{a}=2, \epsilon_{b} / \epsilon_{a}=0.5$, and $x_{a}=0.5$. The GFD theory accurately predicts the compressibility factor for all temperatures at high densities. However, the accuracy of the GFD predictions deteriorates at lower densities with decreasing temperatures. This error can again be traced to the inaccuracies in the perturbation theory for the reference fluids and the

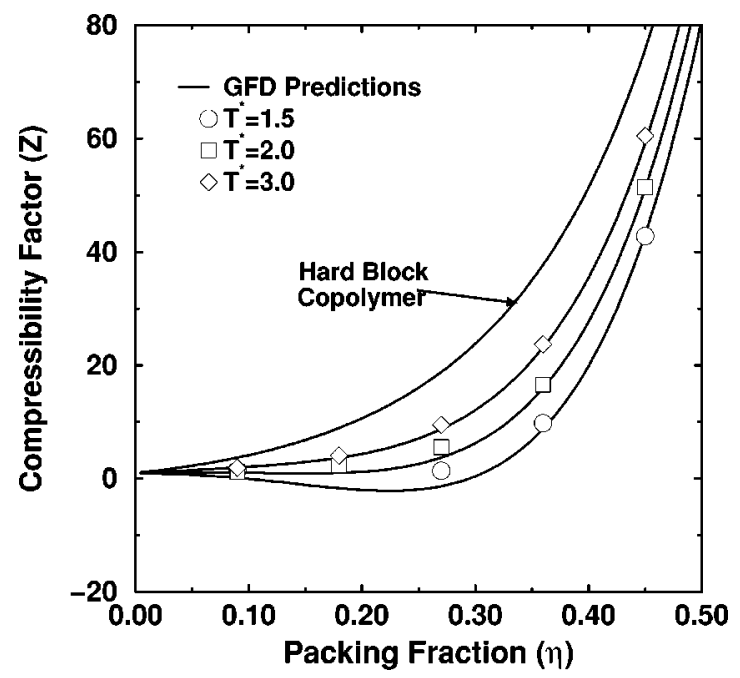

FIG. 6. Compressibility factor vs packing fraction for a 16-mer square-well block copolymer at $T^{*}=1.5,2.0$, and 3.0 where $\sigma_{b} / \sigma_{a}=2.0$ and $\epsilon_{b} / \epsilon_{a}$ $=0.5$.

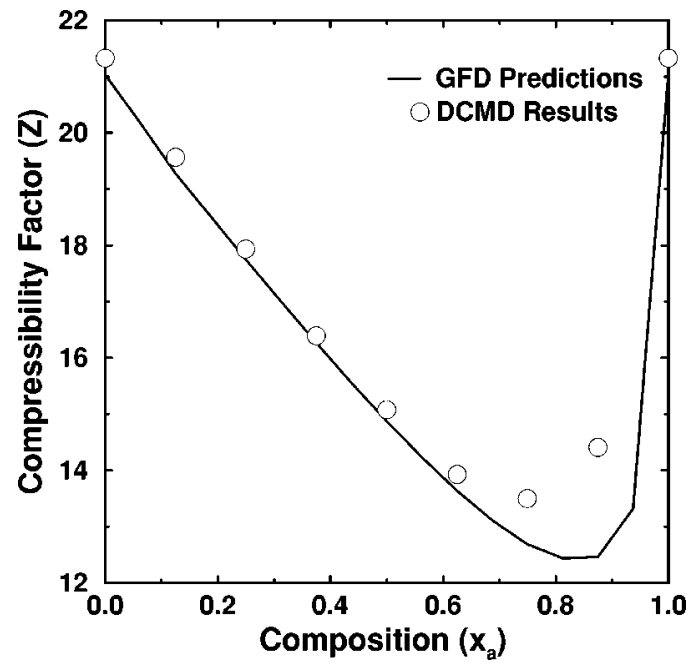

FIG. 7. Compressibility factor vs composition $x_{a}$ for a 16-mer square-well block-copolymer at $\sigma_{b} / \sigma_{a}=2, \epsilon_{b} / \epsilon_{a}=1.0, T^{*}=3.0$, and $\eta=0.36$.

uncertainty in the simulation data. Figure 6 also shows the predictions of the GFD theory for the 16-mer hard (athermal) block copolymer which corresponds to $T^{*}=\infty$. The compressibility factors of the square-well block copolymers asymptotically approach that of a hard-block copolymer with increasing density and increasing temperature. Figure 7 shows the effect of composition on the compressibility factor of a 16-mer square-well block copolymer fluid at $\sigma_{b} / \sigma_{a}$ $=2.0, \epsilon_{b} / \epsilon_{a}=1.0, T^{*}=3$, and $\eta=0.36$. The GFD theory accurately predicts the compressibility factor of the squarewell block copolymer fluid at compositions $x_{a}<0.75$. The accuracy of the GFD theory begins to deteriorate at compositions $x_{a}>0.75$ when the sizes of segments of the two components differ substantially. The source of this error is both geometrical and compositional in origin. In Eq. (15), the insertion factor for the two segments connecting the block was approximated by the insertion factor of a heteronuclear dumbbell in a heteronuclear dumbbell fluid. This approximation gives excellent results for block copolymer fluids where the composition is similar or close to the composition of a heteronuclear dumbbell fluid $\left(x_{a}=0.5\right)$. However, when the composition of the component with larger beads is small, this approximation does not work well.

\section{B. Square-well alternating copolymers}

Figures 8 and 9 compare the GFD predictions to DCMD simulation results for the compressibility factors as a function of $\eta$ for square-well alternating copolymers composed of chains of 4,8 , and 16 segments at reduced temperature $T^{*}=3$. In Fig. 8 the two components have the same hard core diameters $\left(\sigma_{b} / \sigma_{a}=1\right)$ while the relative well-depth is $\epsilon_{b} / \epsilon_{a}=0.5$. In Fig. 9 the ratio of the hard-core diameters of the two components is $\sigma_{b} / \sigma_{a}=2$ and the relative well-depth is $\epsilon_{b} / \epsilon_{a}=0.5$. The predictions of the GFD theory agree well with DCMD simulation results at intermediate densities for all cases. The trends observed in GFD theory for hard alternating copolymer fluids are once again present in GFD theory for square-well alternating copolymer fluids: a slight overestimation of the compressibility factor at very low den- 


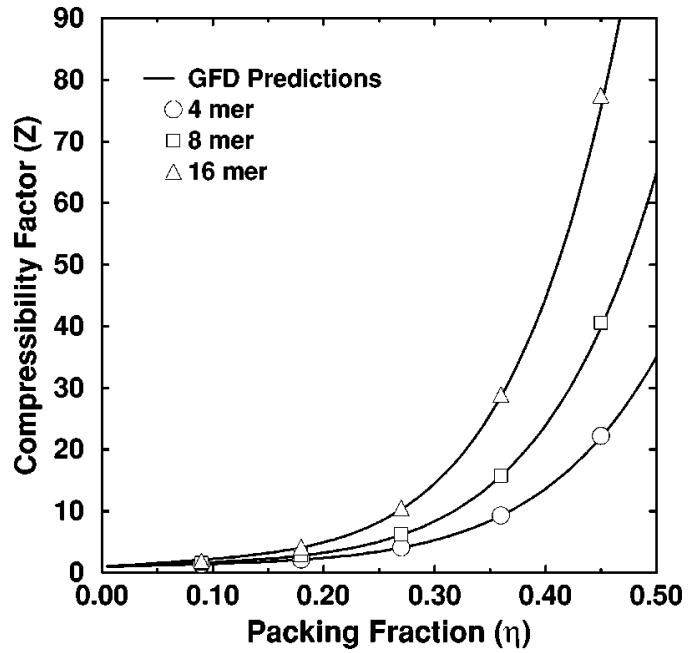

FIG. 8. Square-well alternating-copolymer compressibility factor vs packing fraction for 4-mers, 8-mers, and 16-mers at $T^{*}=3.0$ where $\sigma_{b} / \sigma_{a}=1.0$ and $\epsilon_{b} / \epsilon_{a}=0.5$.

sities and a slight underestimation of the compressibility factor at high densities. Table II summarizes the simulation results and GFD predictions for all square-well alternating copolymer fluids considered.

\section{Square-well random copolymers}

Figures 10 and 11 compare the GFD predictions to DCMD simulation results for the compressibility factors as a function of $\eta$ for square-well random copolymers composed of chains of 8 and 16 segments at reduced temperature $T^{*}=3$. In Fig. 10 the two components have the same hard core diameters $\left(\sigma_{b} / \sigma_{a}=1\right)$ while the relative well-depth is $\epsilon_{b} / \epsilon_{a}=0.5$. In Fig. 11 the ratio of the hard-core diameters of the two component is $\sigma_{b} / \sigma_{a}=2$ and the relative well-depth is $\epsilon_{b} / \epsilon_{a}=0.5$. The GFD theory accurately predicts the compressibility factor of square-well random copolymer fluids over a range of densities and chain lengths. It may be noted that the compressibility factors for 4-mer random copoly-

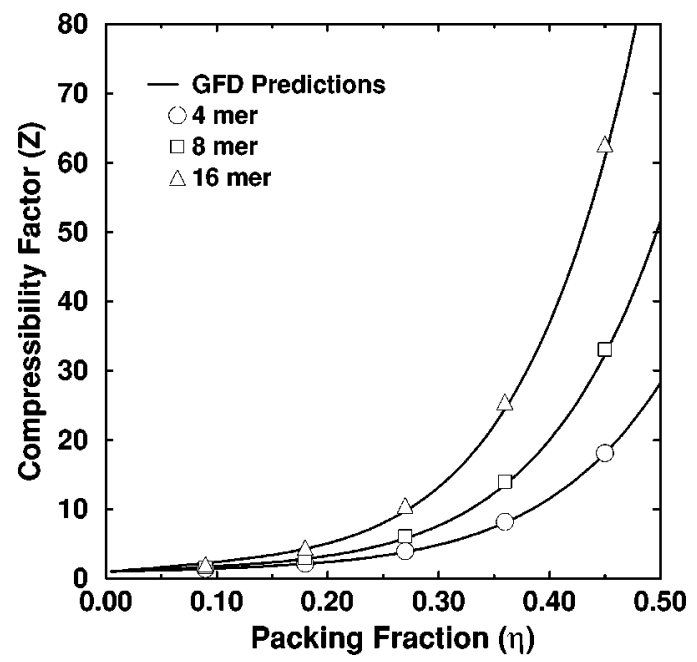

FIG. 9. Square-well alternating-copolymer compressibility factor vs packing fraction for 4-mers, 8-mers, and 16-mers at $T^{*}=3.0$ where $\sigma_{b} / \sigma_{a}=2.0$ and $\epsilon_{b} / \epsilon_{a}=0.5$. mers are not calculated as these chains are too short to use the mathematical probability arguments used to calculate the number of $a a, b b$, and $a b$ pairs on a chain. Along similar lines, the predictions of the GFD theory become more accurate with increasing chain length due to a decrease in the relative error introduced by the probabilistic arguments used to calculate the number of each type of pairs. Table III shows the DCMD simulation results and GFD predictions for all square-well random copolymer fluids considered.

\section{CONCLUSIONS}

In this work we have extended the Generalized FloryDimer theory to copolymer fluids modeled as heteronuclear tangent square-well chain fluids. Expressions for the compressibility factors of square-well block copolymers, squarewell alternating copolymers, and square-well random copolymers have been presented. We have also conducted discontinuous canonical molecular dynamic simulations and report compressibility factor data for square-well block, alternating, and random copolymers containing 4, 8, and 16 segments. The Generalized Flory-Dimer theory for copolymer fluids uses expressions for the insertion factors for square-well monomer mixtures, square-well dimer mixtures, and square-well heteronuclear dumbbells as building blocks. These are obtained from the recently-derived perturbationtheory-based equations of state for square-well monomer mixtures, square-well dimer mixtures, and square-well heteronuclear dumbbells fluids.

The GFD theory accurately predicts the compressibility factor for all three types of square-well copolymer fluids at intermediate densities. The GFD theory slightly overestimates the compressibility factor at low densities and slightly underestimates the compressibility factor at high densities. These trends are consistent with the behavior of the GFD theory for hard chain copolymer fluids and reflect the limitations of the mean field arguments in the GFD theory. The GFD theory is also less accurate at low temperatures. This error at low temperature can partly be attributed to the inaccuracy in the perturbation theory at these state points. We have also studied the effect of temperature and composition on the compressibility factor for block copolymer fluids. The GFD theory accurately predicts the compressibility factor for a range of temperatures and compositions except at very low temperatures or when the composition of the component with smaller beads is very large.

\section{ACKNOWLEDGMENTS}

This work was supported by the Director, Office of Energy Research, Office of Basic Sciences, Chemical Sciences Division of the U.S. Department of Energy under Contract No. DE-FG05-91ER1481. Acknowledgment is made to the Donors of the Petroleum Research Fund, administered by the American Chemical Society, for partial support of this research. 
TABLE II. DCMD simulation values of compressibility factors for square-well alternating copolymers. The value in parentheses represents one standard deviation in the last two significant digits.

\begin{tabular}{|c|c|c|c|c|c|c|c|c|c|}
\hline \multirow[b]{2}{*}{$T^{*}$} & \multirow{2}{*}{$\frac{\sigma_{b}}{\sigma_{a}}$} & \multirow{2}{*}{$\frac{\epsilon_{b}}{\epsilon_{a}}$} & \multirow[b]{2}{*}{$\eta$} & \multicolumn{2}{|c|}{ 4-mer } & \multicolumn{2}{|c|}{ 8-mer } & \multicolumn{2}{|c|}{ 16-mer } \\
\hline & & & & $\mathrm{Z}(\mathrm{MD})$ & $\mathrm{Z}(\mathrm{GFD})$ & $\mathrm{Z}(\mathrm{MD})$ & $\mathrm{Z}(\mathrm{GFD})$ & $\mathrm{Z}(\mathrm{MD})$ & $\mathrm{Z}(\mathrm{GFD})$ \\
\hline \multirow[t]{5}{*}{3} & 1 & 0.5 & 0.09 & $1.351(04)$ & 1.382 & $1.563(11)$ & 1.615 & $1.920(18)$ & 2.080 \\
\hline & & & 0.18 & $2.131(12)$ & 2.099 & $2.844(25)$ & 2.744 & $4.224(30)$ & 4.036 \\
\hline & & & 0.27 & 4.094(17) & 4.014 & $6.263(18)$ & 6.045 & $10.519(49)$ & 10.109 \\
\hline & & & 0.36 & $9.280(11)$ & 9.173 & $15.803(29)$ & 15.521 & $28.881(61)$ & 28.217 \\
\hline & & & 0.45 & $22.210(25)$ & 21.781 & $40.588(63)$ & 39.417 & $77.495(74)$ & 74.689 \\
\hline \multirow[t]{5}{*}{3} & 1 & 1.5 & 0.09 & $0.835(07)$ & 0.913 & $0.702(08)$ & 0.777 & $0.474(32)$ & 0.504 \\
\hline & & & 0.18 & $0.967(08)$ & 0.829 & 0.805 & $0.354(37)$ & $0.524(49)$ & -0.596 \\
\hline & & & 0.27 & $1.860(14)$ & 1.658 & 2.095 & $1.457(54)$ & $2.560(35)$ & 1.054 \\
\hline & & & 0.36 & $5.758(34)$ & 5.804 & 8.855 & $8.780(38)$ & $14.993(74)$ & 14.733 \\
\hline & & & 0.45 & $18.461(28)$ & 18.050 & $32.965(42)$ & 31.706 & $62.016(95)$ & 59.019 \\
\hline \multirow[t]{5}{*}{3} & 2 & 1 & 0.09 & $1.018(10)$ & 1.112 & $1.019(05)$ & 1.145 & $1.033(25)$ & 1.210 \\
\hline & & & 0.18 & $1.301(15)$ & 1.305 & $1.458(19)$ & 1.294 & $1.710(42)$ & 1.270 \\
\hline & & & 0.27 & $2.380(18)$ & 2.291 & $3.149(39)$ & 2.786 & $4.659(69)$ & 3.776 \\
\hline & & & 0.36 & $5.876(23)$ & 5.855 & $9.420(58)$ & 9.078 & $16.460(80)$ & 15.524 \\
\hline & & & 0.45 & $15.631(45)$ & 15.557 & $27.918(93)$ & 27.081 & $52.490(89)$ & 50.128 \\
\hline \multirow[t]{5}{*}{3} & 2 & 0.5 & 0.09 & $1.392(14)$ & 1.417 & $1.627(12)$ & 1.689 & $2.068(09)$ & 2.232 \\
\hline & & & 0.18 & $2.185(25)$ & 2.134 & $2.976(19)$ & 2.849 & $4.479(49)$ & 4.278 \\
\hline & & & 0.27 & $3.967(19)$ & 3.821 & $6.112(34)$ & 5.753 & $10.574(82)$ & 9.617 \\
\hline & & & 0.36 & $8.202(39)$ & 8.036 & $14.006(55)$ & 13.418 & $25.547(44)$ & 24.184 \\
\hline & & & 0.45 & $18.138(32)$ & 17.967 & $33.071(72)$ & 32.036 & $62.747(99)$ & 60.174 \\
\hline
\end{tabular}

\section{APPENDIX A: EXCLUDED VOLUME EXPRESSIONS FOR HETERONUCLEAR CHAINS}

Here we briefly present the expressions used to calculate the various excluded volumes that appear in the GFD theories for hard and square-well block, alternating, and random copolymers.

The GFD equation of state for block copolymer fluids requires knowledge of the volume excluded by a chain (or a block) to a monomer. When the diameter of the chain segment, $\sigma_{\alpha}$, is greater than or equal to the diameter of the monomer, $\sigma_{\beta}$, the excluded volume of a chain is a linear

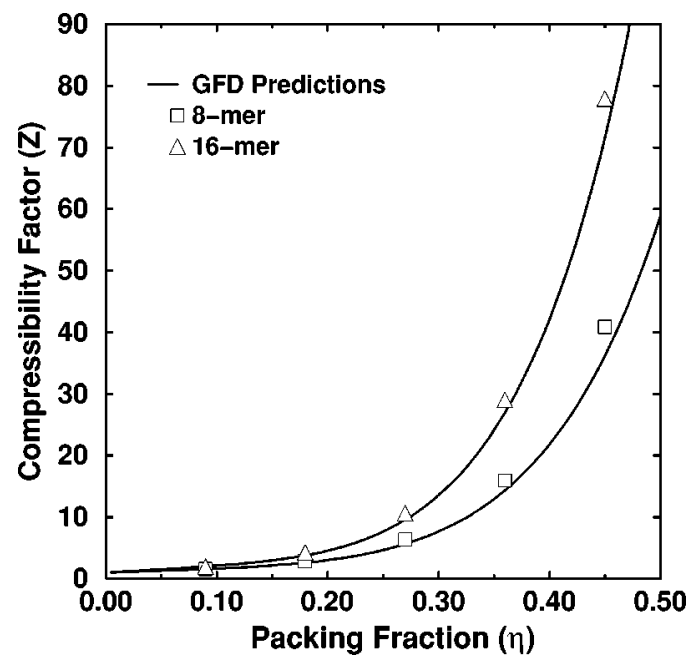

FIG. 10. Square-well random-copolymer compressibility factor vs packing fraction for 8-mers and 16-mers at $T^{*}=3.0$ where $\sigma_{b} / \sigma_{a}=1.0, \epsilon_{b} / \epsilon_{a}$ $=0.5$, and $x_{a}=0.5$. function of the chain length, $n_{\alpha}$, and is accurately fit by the expression

$\left(v_{e}^{\alpha}\left(n_{\alpha}\right)\right)_{\beta}=\left(v_{e}^{\alpha}(3)\right)_{\beta}+\left(n_{\alpha}-3\right)\left[\left(v_{e}^{\alpha}(3)\right)_{\beta}-\left(v_{e}^{\alpha}(2)\right)_{\beta}\right]$,

where $\left(v_{e}^{\alpha}(2)\right)_{\beta}$ and $\left(v_{e}^{\alpha}(3)\right)_{\beta}$ are the volumes excluded by a dimer and a trimer of component $\alpha$ to a monomer of component $\beta$. The dimer excluded volume is given by

$$
\left(v_{e}^{\alpha}(2)\right)_{\beta}=\frac{\pi \sigma_{\alpha}^{3}}{6}\left[2+6\left(\frac{\sigma_{\beta}}{\sigma_{\alpha}}\right)+4.5\left(\frac{\sigma_{\beta}}{\sigma_{\alpha}}\right)^{2}+\left(\frac{\sigma_{\beta}}{\sigma_{\alpha}}\right)^{3}\right] .
$$

The trimer excluded volume is empirically calculated to be

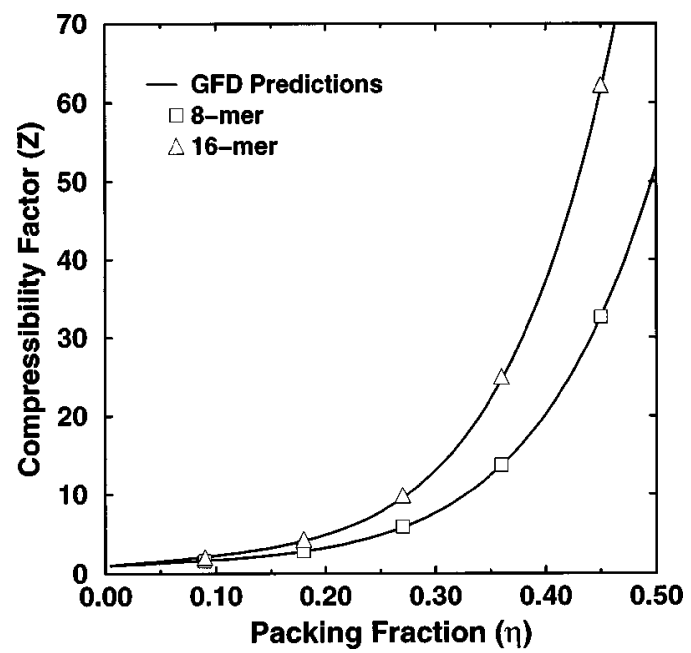

FIG. 11. Square-well random-copolymer compressibility factor vs packing fraction for 8-mers and 16-mers at $\sigma_{b} / \sigma_{a}=2.0, \epsilon_{b} / \epsilon_{a}=0.5$, and $x_{a}=0.5$. 
TABLE III. DCMD simulation values of compressibility factors for squarewell random copolymers. The value in parentheses represents one standard deviation in the last two significant digits.

\begin{tabular}{|c|c|c|c|c|c|c|c|c|}
\hline \multirow[b]{2}{*}{$T^{*}$} & \multirow[b]{2}{*}{$\frac{\sigma_{b}}{\sigma_{a}}$} & \multirow[b]{2}{*}{$\frac{\epsilon_{b}}{\epsilon_{a}}$} & \multirow[b]{2}{*}{$x_{a}$} & \multirow[b]{2}{*}{$\eta$} & \multicolumn{2}{|c|}{ 8-mer } & \multicolumn{2}{|c|}{ 16-mer } \\
\hline & & & & & $\mathrm{Z}(\mathrm{MD})$ & Z(GFD) & Z(MD) & $\mathrm{Z}(\mathrm{GFD})$ \\
\hline \multirow[t]{5}{*}{3} & 1 & 0.5 & 0.5 & 0.09 & $1.567(15)$ & 1.575 & $1.942(14)$ & 2.014 \\
\hline & & & & 0.18 & $2.853(10)$ & 2.612 & $4.283(07)$ & 3.825 \\
\hline & & & & 0.27 & $6.339(13)$ & 5.658 & $10.637(42)$ & 9.622 \\
\hline & & & & 0.36 & $15.940(31)$ & 14.333 & $28.984(72)$ & 26.953 \\
\hline & & & & 0.45 & $40.851(36)$ & 36.047 & $77.906(35)$ & 71.283 \\
\hline \multirow[t]{5}{*}{3} & 2 & 0.5 & 0.5 & 0.09 & $1.606(37)$ & 1.677 & $2.051(17)$ & 2.171 \\
\hline & & & & 0.18 & $2.885(42)$ & 2.857 & $4.316(24)$ & 4.177 \\
\hline & & & & 0.27 & $5.948(53)$ & 5.856 & $9.906(13)$ & 9.623 \\
\hline & & & & 0.36 & $13.740(91)$ & 13.705 & 24.994(53) & 24.586 \\
\hline & & & & 0.45 & $32.650(87)$ & 32.600 & $62.265(89)$ & 61.501 \\
\hline
\end{tabular}

$\left(v_{e}^{\alpha}(3)\right)_{\beta}=\sigma_{\alpha}^{3}\left[1.57+4.75\left(\frac{\sigma_{\beta}}{\sigma_{\alpha}}\right)+2.99\left(\frac{\sigma_{\beta}}{\sigma_{\alpha}}\right)^{2}+0.52\left(\frac{\sigma_{\beta}}{\sigma_{\alpha}}\right)^{3}\right]$.

When the diameter of the chain segment is smaller than the diameter of the monomer $\left(\sigma_{\alpha}<\sigma_{\beta}\right)$, the excluded volume between the chain and monomer is accurately fit by the expression

$$
\begin{aligned}
\left(v_{e}^{\alpha}\left(n_{\alpha}\right)\right)_{\beta}= & \sigma_{\alpha}^{3}\left[1.67\left(\frac{\sigma_{\beta}}{\sigma_{\alpha}}\right)^{2.543}+2.594 n_{\alpha}\left(\frac{\sigma_{\beta}}{\sigma_{\alpha}}\right)^{1.241}\right. \\
& \left.-0.00041 n_{\alpha}^{2}\left(\frac{\sigma_{\beta}}{\sigma_{\alpha}}\right)^{3.165}\right] .
\end{aligned}
$$

The GFD equation of state for alternating copolymer fluids requires knowledge of the volume excluded by alternating trimers. The volume excluded by an $\alpha \beta \alpha$ trimer to a monomer of component $\alpha$ is conveniently fit by the expression

$$
\begin{aligned}
\left(v_{e}^{\alpha \beta \alpha}(3)\right)_{\alpha}= & \sigma_{\alpha}^{3}\left[7.13+0.3387\left(\frac{\sigma_{\beta}}{\sigma_{\alpha}}\right)+1.841\left(\frac{\sigma_{\beta}}{\sigma_{\alpha}}\right)^{2}\right. \\
& \left.+0.507\left(\frac{\sigma_{\beta}}{\sigma_{\alpha}}\right)^{3}\right]
\end{aligned}
$$

for $0.4<\sigma_{\beta} / \sigma_{\alpha}<2.5$. The volume excluded by an $\alpha \beta \alpha$ trimer to a monomer of component $\beta$ is fit by the expression

$$
\begin{aligned}
\left(v_{e}^{\alpha \beta \alpha}(3)\right)_{\beta}= & \sigma_{\alpha}^{3}\left[0.911+3.956\left(\frac{\sigma_{\beta}}{\sigma_{\alpha}}\right)+0.93\left(\frac{\sigma_{\beta}}{\sigma_{\alpha}}\right)^{2}\right. \\
& \left.+4.026\left(\frac{\sigma_{\beta}}{\sigma_{\alpha}}\right)^{3}\right] .
\end{aligned}
$$

The volume excluded by a trimer of type $\beta \alpha \beta$ can also be calculated from the above two expressions by interchanging the indices.

The GFD equation of state for a random copolymer requires knowledge of the volumes excluded by the trimers $\alpha \alpha \alpha, \alpha \beta \alpha$, and $\alpha \beta \beta$. While the expressions for the volumes excluded by trimers $\alpha \alpha \alpha$ and $\alpha \beta \alpha$ are given in Eqs.
(A3), (A5) and (A6), the volume excluded by a heteronuclear trimer $\alpha \beta \beta$ to monomers of components $\alpha$ and $\beta$ can be fitted by the following expressions:

$$
\begin{aligned}
\left(v_{e}^{\alpha \beta \beta}(3)\right)_{\alpha}= & \sigma_{\alpha}^{3}\left[3.86+1.64\left(\frac{\sigma_{\beta}}{\sigma_{\alpha}}\right)+3.28\left(\frac{\sigma_{\beta}}{\sigma_{\alpha}}\right)^{2}\right. \\
& \left.+1.038\left(\frac{\sigma_{\beta}}{\sigma_{\alpha}}\right)^{3}\right]
\end{aligned}
$$

and

$$
\begin{aligned}
\left(v_{e}^{\alpha \beta \beta}(3)\right)_{\beta}= & \sigma_{\alpha}^{3}\left[-1.025+7.53\left(\frac{\sigma_{\beta}}{\sigma_{\alpha}}\right)-5.475\left(\frac{\sigma_{\beta}}{\sigma_{\alpha}}\right)^{2}\right. \\
& \left.+8.77\left(\frac{\sigma_{\beta}}{\sigma_{\alpha}}\right)^{3}\right] .
\end{aligned}
$$

It may be noted that the fits given in Eqs. (A5) through (A8) differ slightly from their counterparts in Ref. 8. The new fits were obtained by regressing the trimer volume vs diameter ratio data over a larger range of diameter ratios.

\section{APPENDIX B: SQUARE-WELL INSERTION FACTORS FROM PERTURBATION THEORY}

In this appendix, we briefly describe the perturbationtheory-based calculation of the insertion factors for each species in a square-well monomer mixture, $p_{1}^{i}(\eta)_{\mathrm{sw}}$, in a square-well dimer mixture, $p_{2}^{i}(\eta)_{\mathrm{sw}}$, and in a square-well heteronuclear dumbbell fluid, $p_{2}^{a b}(\eta)_{\mathrm{sw}}$. We also present the related "zeta" expressions, $\zeta_{1, \mathrm{sw}}^{i}, \zeta_{2, \mathrm{sw}}^{i}$, and $\zeta_{2, \mathrm{sw}}^{a b}$. The reader is referred to Ref. 14 for details of the theory's derivation. In order to keep the notation general (and to be consistent with the notation used in Ref. 14) we use the subscript $n$ to denote the mixture of $n$-mers. The reader should bear in mind, however, that this $n$ is limited to $n=1$ and $n=2$, and should not be confused with the copolymer chain length referred to in the main body of this paper.

The insertion factor $p_{n}^{i}(\eta)$ of species $i$ in an $n$-mer mixture is related to its corrected excess chemical potential by ${ }^{33}$

$$
\mu_{n}^{i^{*}}(\eta) \equiv \mu_{n}^{i^{e x}}(\eta)-\mu_{n}^{i^{e x}}(0)=-k T \ln p_{n}^{i}(\eta),
$$

where the corrected excess chemical potential is defined as the difference between the excess chemical potential of the species at the density of interest and the excess chemical potential of the species at zero density. The corrected excess chemical potential can be calculated from knowledge of the total excess Helmholtz free energy of the mixture

$$
\mu_{n}^{i^{*}}(\eta)=\left[\frac{\partial\left(N A_{n}^{e x}(\eta)\right)}{\partial N_{i}}\right]_{T, V},
$$

where $N$ is the number of molecules in the system, and $N_{i}$ is the number of molecules of species $i$. Here, we briefly review the perturbation theory approach to calculating the excess Helmholtz free energy of square-well mixtures and derive expressions for the $\zeta_{n}^{i}$.

In the perturbation approach, the free energy of a squarewell $n$-mer mixture is expressed as an expansion in the inverse temperature around the free energy of the reference 
TABLE IV. Geometric parameters for convex bodies.

\begin{tabular}{|c|c|c|c|}
\hline Convex body & $\begin{array}{l}\text { Volume } \\
\qquad b\end{array}$ & $\begin{array}{c}\text { Surface area } \\
\qquad s\end{array}$ & $\begin{array}{c}\text { Radius of } \\
\text { curvature }(r)\end{array}$ \\
\hline Monomer & $\frac{\pi \sigma^{3}}{6}$ & $\pi \sigma^{2}$ & $\sigma / 2$ \\
\hline Homonuclear dimer & $\frac{\pi \sigma^{3}}{3}$ & $2 \pi \sigma^{2}$ & $3 \sigma / 4$ \\
\hline Heteronuclear dimer & $\frac{\pi\left(\sigma_{1}^{3}+\sigma_{2}^{3}\right)}{6}$ & $\pi\left(\sigma_{1}^{2}+\sigma_{2}^{2}\right)$ & $\begin{array}{c}\frac{3}{8}\left(\sigma_{1}+\sigma_{2}\right)+ \\
\frac{\left(\sigma_{2}-\sigma_{1}\right)^{2}}{8\left(\sigma_{2}+\sigma_{1}\right)}\end{array}$ \\
\hline
\end{tabular}

hard-sphere mixture. The second-order perturbation theory expansion for the Helmholtz free energy is of the form

$$
\frac{A_{n, \mathrm{sw}}^{e x}}{N k T}=\frac{A_{n, \mathrm{hs}}^{(0)}}{N k T}+\left(\frac{1}{k T}\right) \frac{A_{n, \mathrm{sw}}^{(1)}}{N k T}+\left(\frac{1}{k T}\right)^{2} \frac{A_{n, \mathrm{sw}}^{(2)}}{N k T},
$$

where $A_{n \text {,hs }}^{(0)}$ is the excess Helmholtz free energy of the reference hard $n$-mer mixture, and $A_{n, \mathrm{sw}}^{(1)}$ and $A_{n, \mathrm{sw}}^{(2)}$ are the firstand second-order perturbation contributions to the Helmholtz free energy of the square-well mixture.

The excess Helmholtz free energy of the reference hard $n$-mer mixture can be calculated from knowledge of its compressibility factor using

$$
\frac{A_{n, \mathrm{hs}}^{(0)}(\eta)}{N k T}=\int_{0}^{\eta} \frac{Z_{\mathrm{hs}}^{n}-1}{\eta} d \eta .
$$

The compressibility factor for the reference hard $n$-mer mixture, $Z_{\mathrm{hs}}^{n}$, is obtained using the scaled particle theory equation of state 29,30

$$
Z_{\mathrm{hs}}^{n}=\frac{1}{1-\eta}+\frac{3 \alpha \eta}{(1-\eta)^{2}}+\frac{3 \alpha^{2} \eta^{2}-\alpha^{2} \eta^{3}}{(1-\eta)^{3}},
$$

where $\alpha=\bar{r} s / 3 b$ is the geometric factor and $\bar{r}, s$ and $b$ are the mean radius of curvature, surface area, and volume, respectively, of the reference hard $n$-mer mixture defined as

$$
b=\sum_{i}^{m} x_{i} b_{i}, \quad s=\sum_{i}^{m} x_{i} s_{i}, \quad \bar{r}=\sum_{i}^{m} x_{i} r_{i},
$$

where $x_{i}$ is the mole fraction of component $i$ and $m$ is the number of components in the mixture. Table IV lists the geometric coefficients for a monomer, homonuclear dimer, and a heteronuclear dumbbell. It may be noted that the expression for the radius of curvature of a heteronuclear dumbbell is different from the corresponding expression presented in Ref. 8, which was erroneous.

The first-order perturbation contribution to the Helmholtz free energy is given by ${ }^{14,34,35}$

$$
\frac{A_{n, \mathrm{sw}}^{(1)}}{N k T}=-2 \pi \rho n^{2}\left[\sigma^{3} \epsilon I_{0}\right]_{\mathrm{mix}},
$$

TABLE V. Coefficients for the polynomial expansion of the integral $I_{0}^{i}$ $=\Sigma_{k} c_{k}^{i} \eta^{k}$.

\begin{tabular}{ccc}
\hline \hline$c_{k}$ & Monomer fluid & Dimer fluid \\
\hline$c_{0}$ & 0.716297 & 0.5052058 \\
$c_{1}$ & 1.108746 & 1.054739 \\
$c_{2}$ & 0.059607 & 1.821172 \\
$c_{3}$ & 0.054981 & -1.462742 \\
$c_{4}$ & -9.550753 & -18.976502 \\
$c_{5}$ & 25.31761 & 62.173311 \\
$c_{6}$ & -53.28665 & -108.21679 \\
$c_{7}$ & 43.11756 & 75.093454 \\
& & \\
\hline \hline
\end{tabular}

where $\rho$ is the number density, $n$ is the number of segments per molecule ( $n=1$ for a monomer mixture and $n=2$ for a dimer mixture or a dumbbell fluid), and

$$
\left[\sigma^{3} \epsilon I_{0}\right]_{\mathrm{mix}}=\sum_{i}^{m} \sum_{j}^{m} x_{i} x_{j} \sigma_{i j}^{3} \epsilon_{i j} I_{0}^{i j},
$$

where $\sigma_{i j}=\left(\sigma_{i}+\sigma_{j}\right) / 2$ is the mean diameter of the monomer segments of components $i$ and $j, \epsilon_{i j}$ is defined according to the combining rule in Eq. (2), and

$$
I_{0}^{i j}=\int_{1}^{\lambda} g_{0}^{i j}(x) x^{2} d x,
$$

is the integral over $x\left(=r / \sigma_{i j}\right)$ of the scaled site-site radial distribution function $g_{0}^{i j}$ of a molecule of component $j$ about the molecule of component $i$. It has recently been shown ${ }^{14}$ that even for an $n$-mer mixture where the two components have segments of different size, $I_{0}^{i j}$ can be approximated by $I_{0}^{n}$, the integral of the radial distribution function of an $n$-mer fluid in which all segments are the same size. For example, $I_{0}^{i j}$ for a dimer-mixture, where the segment sizes of the two components differ, can be approximated by $I_{0}^{2}$, the integral of the radial distribution function of a pure dimer fluid at the same density. The integral $I_{0}^{n}$ is fitted to a seventh-order polynomial in $\eta$ of the form

$$
I_{0}^{n}=\sum_{k=0}^{7} c_{k} \eta^{k}
$$

Table $\mathrm{V}$ show the coefficients $c_{k}$ for $n=1$ (monomer fluids) and $n=2$ (dimer and dumbbell fluids).

The second-order perturbation contribution to the Helmholtz free energy is calculated using the local compressibility approximation of Barker and Henderson ${ }^{35}$ and is given by

$$
\frac{A_{n, \mathrm{sw}}^{(2)}}{N k T}=-\pi \rho n^{2}\left[\sigma^{3} \epsilon^{2}\right]_{\text {mix }} \frac{\partial \rho}{\partial p_{0}} \frac{\partial\left(\eta I_{0}^{n}\right)}{\partial \eta},
$$

where

$$
\left[\sigma^{3} \epsilon^{2}\right]_{\mathrm{mix}}=\sum_{i}^{m} \sum_{j}^{m} x_{i} x_{j} \sigma_{i j}^{3} \epsilon_{i j}^{2}
$$


and $p_{0}=\rho k T Z_{0}$ is the pressure of the reference hard system which is obtained using scaled particle theory.

The insertion factor for a species $i$ in a $n$-mer mixture is obtained by inserting the excess Helmholtz free energy from Eqs. (B3), (B4), (B7), and (B11), into Eq. (B2). According to Eq. (B1) the natural logarithm of the insertion factor can be expressed in the form

$$
\ln p_{n, \mathrm{sw}}^{i}=\ln p_{n, \mathrm{hs}}^{i,(0)}+\left(\frac{1}{k T}\right) \ln p_{n}^{i,(1)}+\left(\frac{1}{k T}\right)^{2} \ln p_{n}^{i,(2)}
$$

where $p_{n, \mathrm{hs}}^{i,(0)}$ is the insertion factor for the reference hard system, and $p_{n}^{i,(1)}$ and $p_{n}^{i,(2)}$ are the first- and second-order contributions. Substituting the above general form for $\ln p_{n, \text { sw }}^{i}$ into the definition for $\zeta_{n}^{i}$ [Eq. (20)] and carrying out the integration, we obtain the final expression for the $\zeta_{n, \mathrm{sw}}^{i}$ which can be expressed in the form

$$
\zeta_{n, \mathrm{sw}}^{i}=\zeta_{n, \mathrm{hs}}^{i,(0)}+\left(\frac{1}{k T}\right) \zeta_{n, \mathrm{sw}}^{i,(1)}+\left(\frac{1}{k T}\right)^{2} \zeta_{n, \mathrm{sw}}^{i,(2)}
$$

where $\zeta_{n, \mathrm{hs}}^{i,(0)}$ is the hard-sphere contribution to $\zeta_{n, \mathrm{sw}}^{i}$ and $\zeta_{n, \mathrm{sw}}^{i,(1)}$ and $\zeta_{n, \mathrm{sw}}^{i,(2)}$ are the first- and second-order contributions. The expression for $\zeta_{n, \mathrm{hs}}^{i,(0)}$ has been derived by Gulati and $\mathrm{Hall}^{8}$ and is expressed as

$$
\begin{aligned}
\zeta_{n, \mathrm{hs}}^{i,(0)}= & -\left(1+W_{i}+Y_{i}+3 Z_{i}\right) \ln (1-\eta) / \eta \\
& +\left\{-\left[Y_{i}+3 Z_{i}+W_{i}\right]+\eta\left[-X_{i} / 2+(5 / 2) Y_{i}\right.\right. \\
& \left.+(15 / 2) Z_{i}+3 W_{i}\right]-\eta^{2}\left[X_{i} / 2+(5 / 2) Y_{i}\right. \\
& \left.\left.+(11 / 2) Z_{i}+3 W_{i}\right]+\eta^{3} W_{i}\right\}(1-\eta)^{-3},
\end{aligned}
$$

where

$$
\begin{aligned}
& X_{i}=-S_{1 i}-S_{4 i}, \quad Y_{i}=2 S_{1 i}-S_{2 i}+\frac{5}{2} S_{4 i}, \\
& Z_{i}=S_{2 i}-S_{1 i}-S_{3 i}-\frac{3}{2} S_{4 i}+S_{5 i}, \quad W_{i}=-S_{4 i},
\end{aligned}
$$

and

$$
\begin{aligned}
& S_{1 i}=\frac{\bar{r}_{i} s+s_{i} \bar{r}+b_{i}}{b}, \\
& S_{2 i}=\left[\frac{1}{6} r_{i}^{2} s^{2}+\frac{1}{3} c s s_{i}+b_{i} \bar{r} s\right] / b^{2}, \\
& S_{3 i}=\frac{1}{3} \frac{b_{i} c s^{2}}{b^{3}}, \\
& S_{4 i}=\left[b\left(r_{i}^{2} s^{2}+2 c s s_{i}\right)-2 c s^{2} b_{i}\right] /\left(9 b^{3}\right), \\
& S_{5 i}=c s^{2} b_{i} /\left(9 b^{3}\right), \\
& c=\sum x_{i} r_{i}^{2} .
\end{aligned}
$$

The coefficients $b, s$, and $\bar{r}$ for a mixture that appear in Eqs. (B16)-(B20) are defined in Eq. (B6).

The first-order perturbation term for $\zeta$ is given by

$$
\begin{aligned}
\zeta_{n}^{i,(1)}= & -12 n \sigma_{i}^{3} \frac{\left[\sigma^{3} \epsilon\right]_{\operatorname{mix}}}{\left[\sigma^{3}\right]_{\operatorname{mix}}^{2}}\left[\eta^{2} \frac{\partial I_{0}^{n}}{\partial \eta}-\frac{1}{\eta} \int_{0}^{\eta} \eta^{2} \frac{\partial I_{0}^{n}}{\partial \eta} \mathrm{d} \eta\right] \\
& -\frac{24 n}{\left[\sigma^{3}\right]_{\operatorname{mix}}}\left(x_{i} \sigma_{i}^{3} \epsilon_{i}+\sum_{j \neq i} x_{j} \sigma_{i j}^{3} \epsilon_{i j}\right) \\
& \times\left[\eta I_{0}^{n}-\frac{1}{\eta} \int_{0}^{\eta} \eta I_{0}^{n} \mathrm{~d} \eta\right],
\end{aligned}
$$

where

$$
\left[\sigma^{3} \epsilon\right]_{\operatorname{mix}}=\sum_{i} \sum_{j} x_{i} x_{j} \sigma_{i j}^{3} \epsilon_{i j}, \quad\left[\sigma^{3}\right]_{\operatorname{mix}}=\sum_{i} x_{i} \sigma_{i}^{3} .
$$

The integrals can be evaluated easily due to the simple polynomial form of $I_{0}^{n}$ shown in Eq. (B10), yielding

$$
\begin{aligned}
& \int_{0}^{\eta} \eta I_{0}^{n} \mathrm{~d} \eta=\sum_{k=0}^{7} c_{k} \frac{\eta^{k+2}}{k+2}, \\
& \int_{0}^{\eta} \eta^{2} \frac{\partial I_{0}^{n}}{\partial \eta} \mathrm{d} \eta=\sum_{k=1}^{7} \frac{k}{k+2} c_{k} \eta^{k+2} .
\end{aligned}
$$

The coefficients $c_{k}$ are listed in Table V.

The second-order perturbation contribution to $\zeta_{n}^{i}$ is given by

$$
\begin{aligned}
\zeta_{n}^{i,(2)}= & -6 n \sigma_{i}^{3} \frac{\left[\sigma^{3} \epsilon^{2}\right]_{\operatorname{mix}}}{\left[\sigma^{3}\right]_{\operatorname{mix}}^{2}}\left[\frac{\eta^{2}(1-\eta)^{4}}{F_{1}(\eta)} \frac{\partial^{2}\left(\eta I_{0}^{n}\right)}{\partial \eta^{2}}-\frac{1}{\eta} \int_{0} \frac{\eta^{2}(1-\eta)^{4}}{F_{1}(\eta)} \frac{\partial^{2}\left(\eta I_{0}^{n}\right)}{\partial \eta^{2}} \mathrm{~d} \eta-\frac{\eta^{2}(1-\eta)^{3} F_{2}(\eta)}{F_{1}^{2}(\eta)} \frac{\partial\left(\eta I_{0}^{n}\right)}{\partial \eta}-\frac{1}{\eta}\right. \\
& \left.\times \int_{0}^{\eta} \frac{\eta^{2}(1-\eta)^{3} F_{2}(\eta)}{F_{1}^{2}(\eta)} \frac{\partial\left(\eta I_{0}^{n}\right)}{\partial \eta} \mathrm{d} \eta\right]-\frac{12 n}{\left[\sigma^{3}\right]_{\operatorname{mix}}^{2}}\left(x_{i} \sigma_{i}^{3} \epsilon_{i}^{2}+\sum_{j \neq i} x_{j} \sigma_{i j}^{3} \epsilon_{i j}^{2}\right)\left[\frac{\eta(1-\eta)^{4}}{F_{1}(\eta)} \frac{\partial\left(\eta I_{0}^{n}\right)}{\partial \eta}\right. \\
& \left.-\frac{1}{\eta} \int_{0} \frac{\eta(1-\eta)^{4}}{F_{1}(\eta)} \frac{\partial\left(\eta I_{0}^{n}\right)}{\partial \eta} \mathrm{d} \eta\right],
\end{aligned}
$$


where

$$
F_{1}(\eta)=(1-\eta)^{2}+6 \alpha \eta(1-\eta)-\alpha^{2} \eta^{2}\left(9-4 \eta+\eta^{2}\right)
$$

and

$$
\begin{aligned}
F_{2}(\eta)= & (1-\eta)^{2}+6 \alpha\left(1-\eta^{2}\right) \\
& -\alpha^{2} \eta\left(18-3 \eta+4 \eta^{2}-\eta^{3}\right) .
\end{aligned}
$$

${ }^{1}$ M. S. Wertheim, J. Stat. Phys. 35, 19 (1984).

${ }^{2}$ M. S. Wertheim, J. Chem. Phys. 87, 7323 (1987).

${ }^{3}$ W. G. Chapman, G. Jackson, and K. E. Gubbins, Mol. Phys. 65, 1057 (1988).

${ }^{4}$ A. Chakrabarti and R. Toral, Macromolecules 23, 2016 (1990).

${ }^{5}$ A. Yethiraj and C. K. Hall, J. Chem. Phys. 95, 8494 (1991).

${ }^{6}$ Y. C. Chiew, Mol. Phys. 70, 129 (1990).

${ }^{7}$ Y. Song, S. M. Lambert, and J. M. Prausnitz, Macromolecules 27, 441 (1994).

${ }^{8}$ H. S. Gulati, J. M. Wichert, and C. K. Hall, J. Chem. Phys. 104, 5220 (1996).

${ }^{9}$ K. P. Shukla and W. G. Chapman, Mol. Phys. 91, 1075 (1997).

${ }^{10}$ F. S. Bates, Annu. Rev. Phys. Chem. 41, 525 (1990).

${ }^{11}$ P. J. Flory, J. Chem. Phys. 10, 51 (1942).

${ }^{12}$ M. L. Huggins, Ann. (N.Y.) Acad. Sci. 43, 1 (1942).
${ }^{13}$ A. Yethiraj and C. K. Hall, Mol. Phys. 72, 619 (1991).

${ }^{14}$ H. S. Gulati and C. K. Hall, J. Chem. Phys. 107, 3930 (1997).

${ }^{15}$ H. C. Anderson, J. Chem. Phys. 72, 2384 (1980).

${ }^{16}$ B. J. Alder and T. E. Wainwright, J. Chem. Phys. 31, 459 (1959).

${ }^{17}$ D. C. Rapaport, J. Phys. A 11, L213 (1978).

${ }^{18}$ D. C. Rapaport, J. Chem. Phys. 71, 3299 (1979).

${ }^{19}$ A. Bellemans, J. Orban, and D. V. Belle, Mol. Phys. 39, 781 (1980).

${ }^{20}$ Y. Zhou, C. K. Hall, and M. Karplus, Phys. Rev. Lett. 77, 2822 (1996).

${ }^{21}$ Y. Zhou, M. Karplus, J. M. Wichert, and C. K. Hall, J. Chem. Phys. 107, 10691 (1997).

${ }^{22}$ R. H. Fowler, Statistical Thermodynamics (Cambridge University Press, Cambridge, 1949).

${ }^{23}$ K. G. Honnell and C. K. Hall, J. Chem. Phys. 90, 1841 (1989).

${ }^{24}$ N. F. Carnahan and K. E. Starling, J. Chem. Phys. 51, 635 (1969).

${ }^{25}$ D. J. Tildesley and W. B. Street, Mol. Phys. 41, 85 (1980).

${ }^{26}$ R. Dickman and C. K. Hall, J. Chem. Phys. 85, 4108 (1986).

${ }^{27}$ M. A. Denlinger and C. K. Hall, Mol. Phys. 71, 541 (1990).

${ }^{28}$ W. R. Smith, D. Henderson, and Y. Tago, J. Chem. Phys. 64, 5026 (1976).

${ }^{29}$ T. Boublik, Mol. Phys. 27, 1415 (1974).

${ }^{30}$ T. Boublik, J. Chem. Phys. 63, 4084 (1975).

${ }^{31}$ F. W. Tavares, J. Chang, and S. I. Sandler, Mol. Phys. 86, 1451 (1995).

${ }^{32}$ T. Hino and J. M. Prausnitz, Fluid Phase Equilibria 138, 105 (1997).

${ }^{33}$ S. K. Kumar, I. Szleifer, C. K. Hall, and J. M. Wichert, J. Chem. Phys. 104, 9100 (1996).

${ }^{34}$ J. Chang and S. I. Sandler, Mol. Phys. 81, 745 (1994).

${ }^{35}$ J. A. Barker and D. Henderson, J. Chem. Phys. 47, 2856 (1967). 